\author{
WALDEN \\ UNIVERSITY \\ A higher degree. A higher purpose.
}

Walden University ScholarWorks

Walden Dissertations and Doctoral Studies

2019

\title{
Predicting Risk for Opioid-Induced Sedation and Respiratory Depression in Hospitalized Patients
}

Alison Partridge

Walden University

Follow this and additional works at: https://scholarworks.waldenu.edu/dissertations

Part of the Nursing Commons

This Dissertation is brought to you for free and open access by the Walden Dissertations and Doctoral Studies Collection at ScholarWorks. It has been accepted for inclusion in Walden Dissertations and Doctoral Studies by an authorized administrator of ScholarWorks. For more information, please contact ScholarWorks@waldenu.edu. 


\title{
Walden University
}

\author{
College of Health Sciences
}

This is to certify that the doctoral dissertation by

\begin{abstract}
Alison Partridge
has been found to be complete and satisfactory in all respects, and that any and all revisions required by the review committee have been made.

Review Committee

Dr. Janice Long, Committee Chairperson, Nursing Faculty

Dr. Leslie Hussey, Committee Member, Nursing Faculty

Dr. Martin Schiavenato, University Reviewer, Nursing Faculty
\end{abstract}

The Office of the Provost

Walden University

2019 


\begin{abstract}
Predicting Risk for Opioid-Induced Sedation and Respiratory Depression in Hospitalized

Patients

by

Alison Partridge MSN, RN, CPAN
\end{abstract}

MS, Charleston Southern University, 2012

BS, Charleston Southern University, 2008

Dissertation Submitted in Partial Fulfillment

of the Requirements for the Degree of

Doctor of Philosophy

Nursing

Walden University

August 2019 


\begin{abstract}
Pain assessment and management is a fundamental part of nursing care. Opioids are 1 of the interventions utilized to manage pain within the hospital setting and have a known adverse effect called opioid-induced sedation and respiratory depression (OSRD). The purpose of this quantitative study was to create a prediction model with the known risk factors present on admission, to determine how well they predict OSRD. This served as a first step in the creation of a risk screen tool, supported by the cognitive continuum theory, in understanding the judgment and decision-making process to provide safe care. The combination of factors that most accurately predicted the risk of OSRD in patients on admission to an acute care healthcare institution was determined through a retrospective case control analysis. Risk factors present on admission of a case group of 100 patients who had succumbed to OSRD after an opioid administration were matched and compared to a control group of 100 who did not. A binary logistic regression analysis was used to determine how well age, body mass index, obstructive sleep apnea, pulmonary disease, respiratory disease, renal failure, and no opioid use (i.e., being opioid naïve) predicted OSRD. The presence of pulmonary disease, renal disease, cardiac disease, diabetes, and being opioid naïve most accurately predicted OSRD. Although only pulmonary disease and renal disease were statistically significant, the final model included other factors that increase the odds of OSRD, which are encompassed in the proposed tool for future research. Through understanding the factors that predict OSRD, a screening tool was created that could save lives in hospital institutions and lead to positive social change by supporting clinical decision making and care.
\end{abstract}


Predicting Risk for Opioid-Induced Sedation and Respiratory Depression in Hospitalized

\title{
Patients
}

by

Alison Partridge MSN, RN, CPAN

MS, Charleston Southern University, 2012

BS, Charleston Southern University, 2008

\author{
Dissertation Submitted in Partial Fulfillment \\ of the Requirements for the Degree of \\ Doctor of Philosophy
}

Nursing

Walden University

August 2019 


\section{Dedication}

I am so thankful to God who has blessed me with so much and so many people in my life that have contributed to the completion of this dissertation. First, I would like to thank my mom who has supported me the most by spending some extra time with her grandkids so I could work on papers, projects, and discussion posts along this journey and was always open to having them stop in while mommy wrote. Next, my husband and children who have been so patient, understanding, and supportive of me on this endeavor and have sacrificed a lot to help me reach my goals. This is as much yours as it is mine. I love you all. 


\section{Acknowledgments}

I am so thankful to the team who helped me complete this dissertation. Especially to Dr. Long, who was so patient and knowledgeable in her feedback and available for phone calls to keep me on track! I so appreciate Dr. Hussey and her prompt responses with excellent feedback to help me further refine and clarify.

I would like to thank all my family who listened and supported me when I needed it! I would like to thank my colleagues and friends who acted as sound boards and offered great advice. Lastly, I would like to thank my friends who listened to me talk about my topic repeatedly without complaining! I have had such amazing people in my life who have gotten me this far. 


\section{Table of Contents}

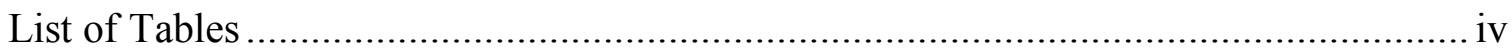

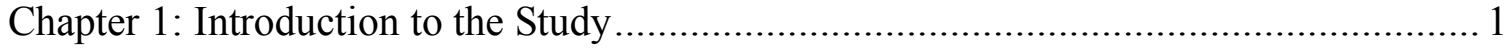

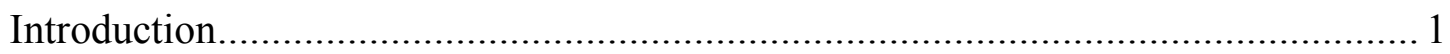

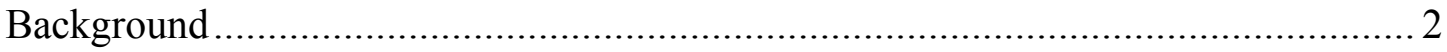

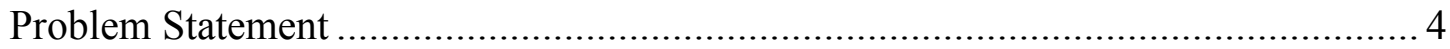

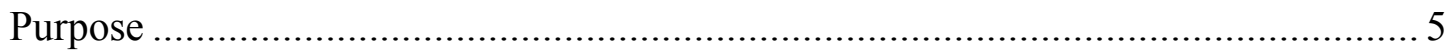

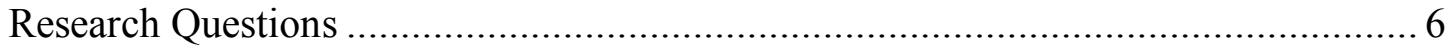

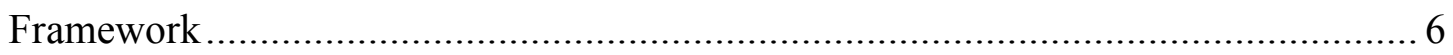

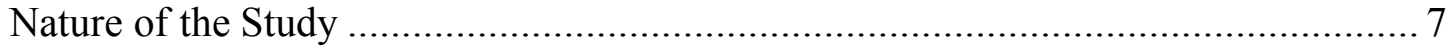

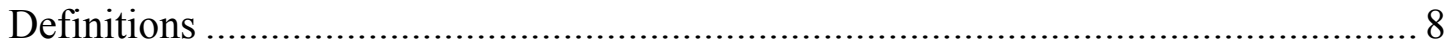

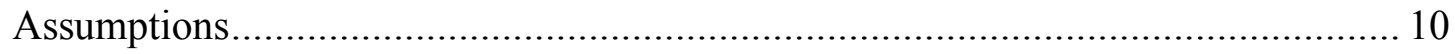

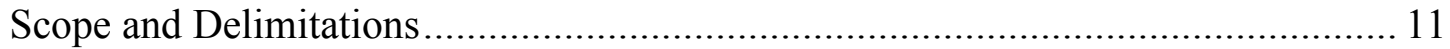

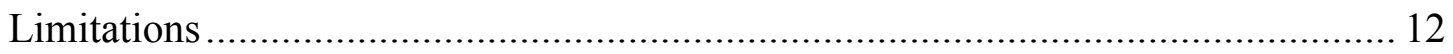

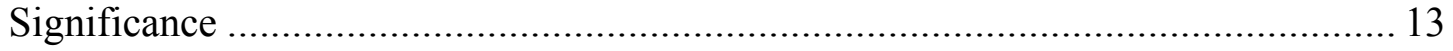

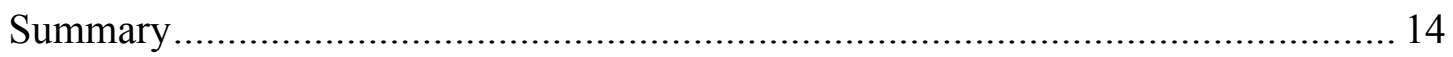

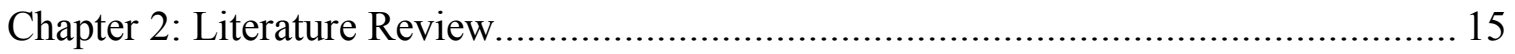

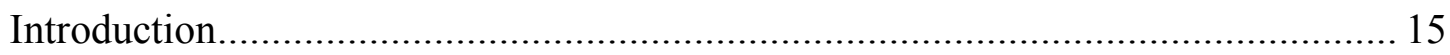

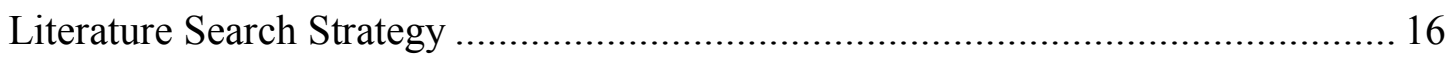

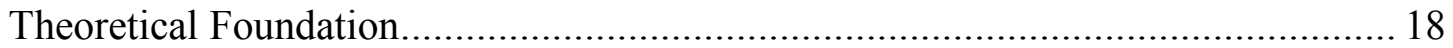

Cognitive Continuum Theory $(\mathrm{CCT})$................................................... 18

Literature Review Related to Key Variables and Concepts .................................. 20 
Synthesis of Studies on the Predictors of OSRD

Opioid-Induced Sedation and Respiratory Depression (OSRD).........................24

Predictors of Opioid-Induced Sedation and Respiratory Depression...................26

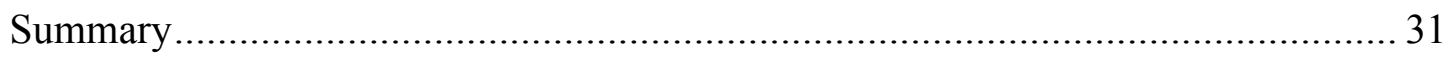

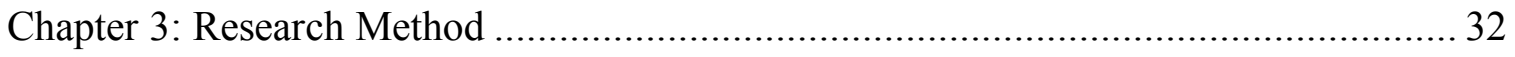

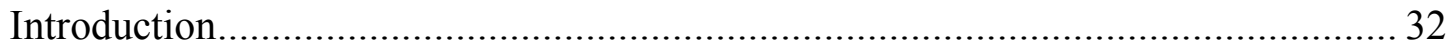

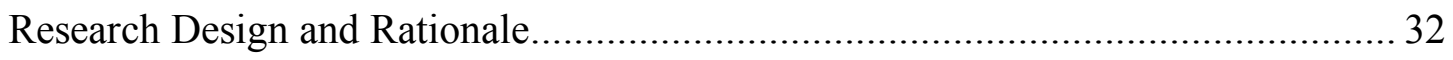

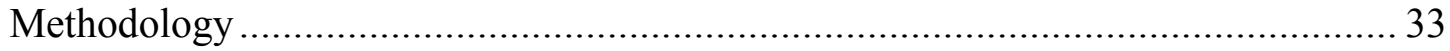

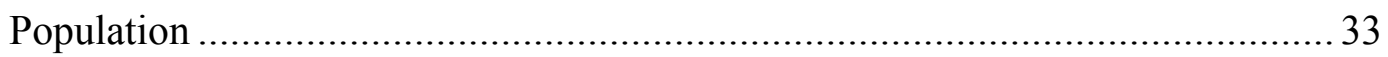

Sampling and Sampling Procedures ..................................................................... 34

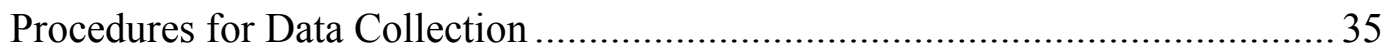

Instrumentation and Operationalization of Constructs .........................................36

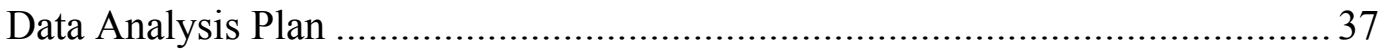

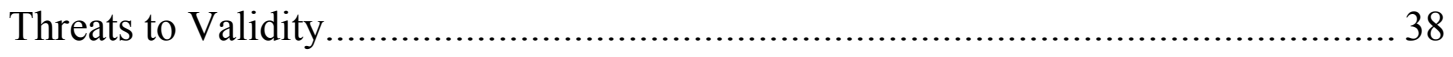

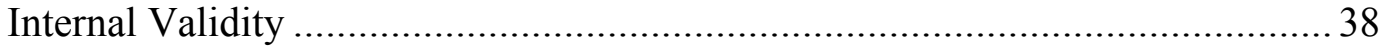

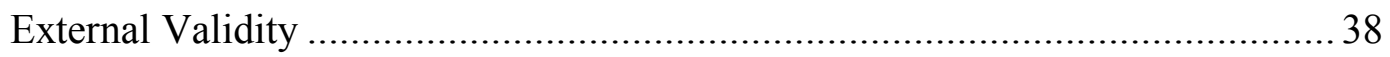

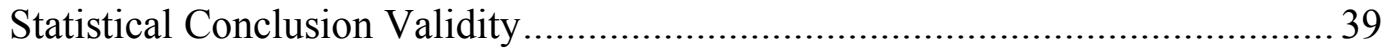

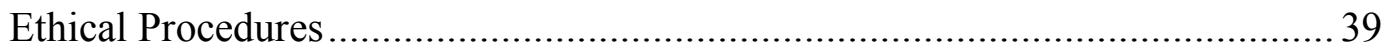

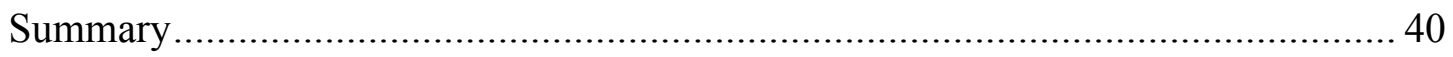

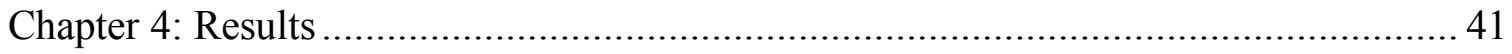

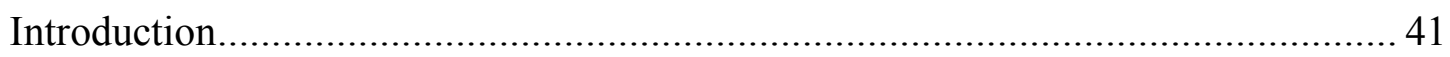

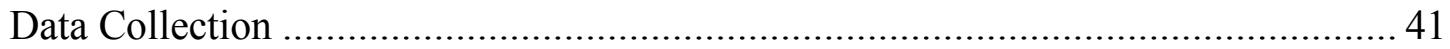




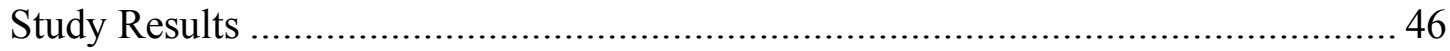

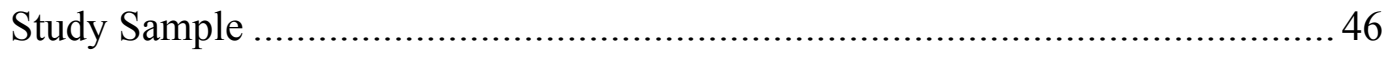

Assumptions of Binary Logistic Regression..................................................... 47

Research Question Results.......................................................................... 49

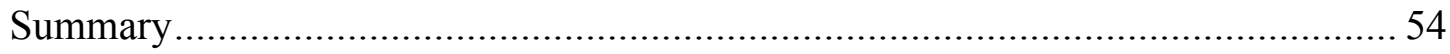

Chapter 5: Discussion, Conclusions, and Recommendations ........................................ 55

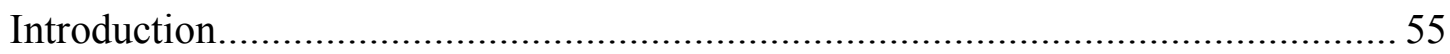

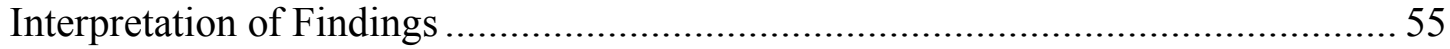

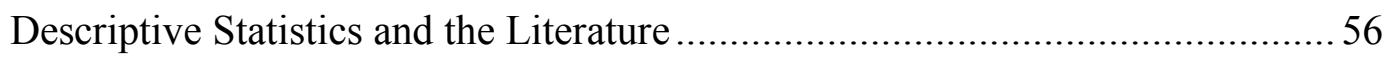

Case-Control Result Comparison .....................................................................5 58

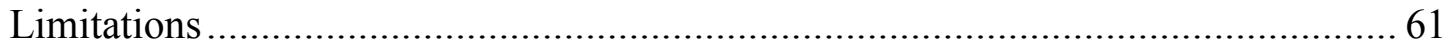

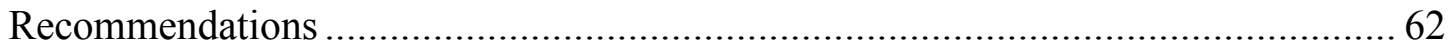

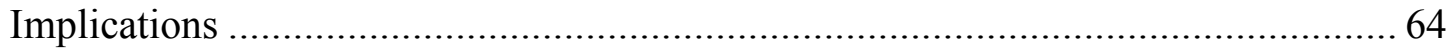

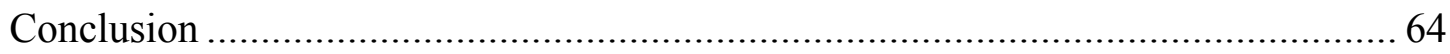

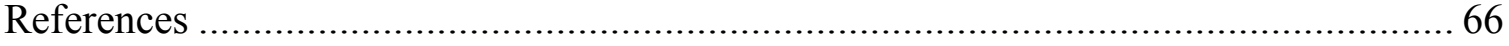

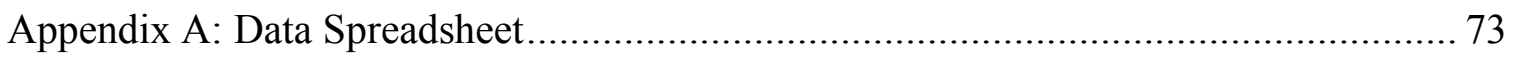

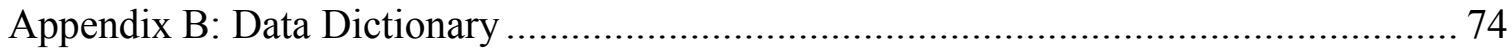

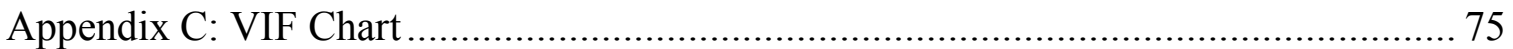




\section{List of Tables}

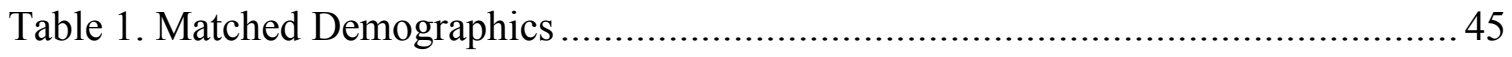

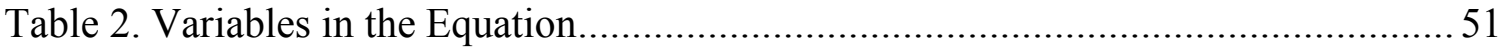

Table 3. Step-Wise Variables in the Equation............................................................52

Table 4. Final Variables in the Equation....................................................................5 
Chapter 1: Introduction to the Study

\section{Introduction}

Pain assessment and management is an integral part of nursing care. Opioids are one of the interventions utilized to manage pain within the hospital setting. A full understanding of the risks of oversedation from opioids is essential when a patient presents to the hospital setting. Publicized as the opioid epidemic, the public is becoming more aware of the dangers of opioids in relation to overdose and addiction (Frasier \& Plescia, 2019). Opioid-induced sedation and respiratory depression (OSRD) is a known adverse effect associated with patients in the hospital setting (Jungquist, Smith, Wiltse Nicely, \& Polomano, 2017). OSRD has other terms with similar meanings that are encompassed within OSRD or are closely related. These terms described in the literature include the following: opioid adverse drug events, opioid-induced respiratory depression (OIRD), opioid-induced oversedation, and opioid-induced ventilatory impairment (OIVI). The alternate terms are all similarly explained as difficulty arousing or decreased respiratory rate after administration of an opioid, often requiring reversal with an opioid antagonist, Naloxone (Rosenfeld et al., 2015). For this research, OSRD encompassed opioid adverse drug events, OIRD, opioid-induced sedation, and OIVI and was the primary descriptor, measured by the administration of Naloxone.

In the hospital setting, OSRD events are reported by The Joint Commission (TJC; 2012) as occurring in $0.5 \%$ of postsurgical patients, with this estimation thought to be low. TJC has suggested screening; however, there is no standardized method for predicting risk for OSRD. The risk factors for OSRD include: (a) age greater than 55 
years, (b) obesity, (c) untreated obstructive sleep apnea, (d) preexisting

pulmonary/cardiac disease or dysfunction, (e) major organ failure, (f) being a smoker, (g) American Society of Anesthesiologists (ASA) Patient Status Classification of 3 to 5, (h) being opioid naïve; (i) being opioid tolerant and given more than their usual amount, (j) high dose patient controlled analgesia; (k) in the first 24 hours of opioid therapy, (l) the presence of thoracic and other large incisions that may interfere with adequate ventilation, (m) concomitant administration of sedating agents, and (n) patients who have already received Naloxone for opioid reversal (Jungquist et al., 2017). While these factors can be used as a guide for assessment, not knowing how well each factor predicts ORSD limits the ability to accomplish this accurately. No scoring tool has been developed or tested that predicts the risk of OSRD on admission, which would allow nurses to accurately assess risk and provide appropriate interventions to manage risk and optimum pain management and monitoring. A screening tool based on the predictors of OSRD could assist clinicians in pain assessment and management. The first step in developing such a tool was to quantify the predictors for ORSD based on the factors in the literature. In this chapter, I provide a background of OSRD as well as explain the problem and purpose of the study. I also describe the research questions, theoretical underpinning, definitions, assumptions, scope, limitations, and significance of determining the factors that most predict OSRD.

\section{Background}

While healthcare providers are focused on adequate pain management, the challenge is providing pain management while preventing adverse events, such as 
oversedation and respiratory depression. Opioid medications, commonly prescribed for pain, place the patient at risk for oversedation and respiratory depression (TJC, 2012). Assessing risk is essential in mitigating potential harm caused by any medication and needs to be included in the medication administration process (Centers for Medicare \& Medicaid Services, 2014).

Several studies have been conducted to assess predicting factors associated with OSRD, finding many overlapping risk factors, with some having more evidence supporting their contribution to risk over others. Most recently, Gupta et al. (2018) provided a review of 13 articles, stating the incidence of the adverse event of oversedation and respiratory depression in surgical patients ranged from $0.1 \%$ to $23.77 \%$. They also listed several factors that increase a patient's risk, such as age over 65, ASA of a 3 or 4, obstructive sleep apnea (OSA), chronic obstructive lung disease (COPD), cardiac disease, neurological disease, diabetes mellitus, female sex, hypertension, use of patient-controlled analgesia, using sedatives in addition to opioids, and giving opioids by varying routes and from varying prescribers. Minkowitz, Gruschkus, Shah, and Raju (2014) conducted a large retrospective cohort study and analyzed historical risk factors associated with an opioid-related adverse drug event of 6,285 surgical patients. They defined a broader range of opioid-related adverse events to include more than oversedation and respiratory depression, like ileus, pruritus, and constipation, which may explain why some of their risk factors were different than those in the literature linked to only oversedation. The differing risk factors included male gender, regional enteritis, diverticulitis, and ulcerative colitis, and the risk factors that are the same were age, prior 
opioid use, COPD, and cardiac dysrhythmias (Minkowitz et al., 2014). Junquist et al. (2017) discussed the need for identifying patients at-risk for OSRD along with listing the factors recognized as increasing risk initially published by McCaffery and Pasero (2011). These factors have influenced many of the publications on risk factors for OSRD to date, including TJC's (2012) recommendation that highlighted the first 24 hours postsurgery, OSA, increased body mass index, prior experience with opioids, and preexisting chronic pain as high-risk factors.

No tool for screening to predict and stratify patients by their level of risk for OSRD has been published, and this represented the gap in the literature that I sought to fill with this study. The lack of effective means for screening and identifying patients at risk places a burden on patients, their families, and the health system. The financial costs of one serious ORSD event can be millions of dollars per claim and with incidences of OSRD up to $23 \%$ (Junquist et al., 2017). While financial consideration is necessary, patients' lives are more important, and identifying potential at-risk individuals based on predictive factors can assist in instituting appropriate administration and monitoring practices to prevent harm (Junquist et al., 2017).

\section{Problem Statement}

Tools, developed as a method of predicting negative outcomes like OSRD, can inform nursing clinical decision-making, creating a streamlined process for the assessment of critical factors (Junquist el al, 2018). Risk factors associated with OSRD have been retrospectively analyzed, published, and recommended to assess by many nursing and accrediting bodies (TJC, 2012). To appropriately follow guidelines aimed at 
improving patient safety, the risk factors available in the literature should be combined and validated as a tool that is easy to use. Many of the factors listed in the practice recommendation of the TJC (2012) continue to be supported by clinical evidence (e.g., Gupta et al (2018) analyzed multiple retrospective reviews, listing many of the same factors). Although the TJC recommended risk stratification for patients, clinicians do not have a standardized tool to complete the task based on the predictability of the variables that lead to ORSD. There is a gap in the literature because of the variability in evidence of the factors and the lack of an existing tool based on the predictors of OSRD. In this study, I sought to accomplish the first step in creating a tool by understanding which combination of published risk factors most predict OSRD to support clinical decisionmaking in the care of patients receiving opioids for pain management.

\section{Purpose}

While the risk factors for OSRD are available in the literature (Jarzyna et al., 2011; TJC, 2012; Jungquist et al., 2017), no scoring tool has been developed or tested to use for screening patients based on the risk factors that most predict OSRD. The purpose of this quantitative study was to create a prediction model based on published risk factors that are present on admission, which serve as the independent variables and are supported by the most current evidence. Independent variables for this study included OSA, obesity, age, pulmonary disease, cardiac disease, major organ failure, diabetes mellitus, and not currently taking opioids. The dependent variables were patients who had succumbed to OSRD and those who had not after receiving an opioid. After this initial study, I created a tool based on the predictive model to be further tested in subsequent studies. If an OSRD 
screening tool were available, it could provide a means to recognize the need for appropriate interventions to manage risk and provide optimum pain management and monitoring.

\section{Research Questions}

Research Question: What combination of factors most accurately predict the risk of OSRD in patients on admission to an acute care healthcare institution?

$H_{0}$ : The independent variables, which included the continuous variables of obesity, measured in BMI and age, and categorical variables as diagnosed yes or no, which included OSA, pulmonary disease, cardiac disease, major organ failure, diabetes mellitus, and no opioid exposure, do not predict the risk of OSRD.

$H_{\mathrm{A}}$ : The independent variables, which included the continuous variables of obesity, measured in BMI and age, and categorical variables as diagnosed yes or no, which included OSA, pulmonary disease, cardiac disease, major organ failure, diabetes mellitus, and no opioid exposure predict the risk of OSRD.

\section{Framework}

Completing a patient history assessment is essential to planning nursing interventions and decision-making. A simplified tool that evaluates risk factors to predict OSRD can assist in quick analysis and decision-making when managing pain with opioids. The cognitive continuum theory (CCT), introduced by Hammond (1981), is a theory that underpins the judgment and decision-making process. Hammond developed 
the CCT by extending the work of another psychology theorist, Brunswikv (Dhami \& Mumpower, 2018). Analysis and decision-making are crucial for every individual, but in the delivery of nursing care, judgment and decision-making are key to providing safe, effective, competent care.

The CCT offers a theoretical framework for the processes involved in human thinking from a psychological perspective. Cader, Campbell, and Watson (2005) stated, "The more structured a task is, the more analytically induced will be the decision-making mode" (p. 399). Tools provide structure to support well-structured tasks. Per Hammond (1981), tasks are well-structured as opposed to ill-structured; however, both types of tasks are relevant in nursing and described in the CCT (Cader, et al., 2005). Intuition is also essential for clinical decision-making in practice and should be used in conjunction with the CCT (Cader, et al., 2005). Although a tool can assist and support decisions, nurses are still accountable and should use a complete patient assessment along with their clinical judgment to provide care to patients. In Chapter 2, I present more detail on the CCT.

\section{Nature of the Study}

In this study, I gathered quantitative data through a retrospective chart review with case-control analysis, based on the risk factors for OSRD the literature supports. Patient records were reviewed using a medical record-storing software. The evaluation of charts was conducted on patients who received Naloxone for opioid reversal for their pertinent medical history that related to the risk factors of OSRD for this study. The control group was retrieved through chart review and matched based on admitting diagnosis or surgical procedure and gender. I then developed the model and tested it with 
logistic regressions (see Houser, 2015). Logistic regression helped me determine which risk factors, evidenced in the literature, were most predictive for OSRD.

\section{Definitions}

Historical factors: Demographic factors or diseases that a patient has before the surgical procedure or hospitalization, which often do not change throughout the stay unless there is a new diagnosis. In this study, I separated these factors from the practice factors that the TJC (2012) combined in their compilation. The demographic and comorbid factors considered as historical risk factors for OSRD are age, BMI, OSA or symptoms of OSA, pulmonary disease, cardiac disease, major organ failure, smoker, ASA patient classification, diabetes mellitus, COPD, opioid dependence, or no exposure to opioids (TJC, 2012).

Opioid-induced respiratory depression (OIRD): When the respiratory rate and depth is reduced below the patient's baseline after an opioid (Pasero \& McCaffery, 2011). While rates at less than eight breaths a minute are considered severe (Dahan, Aarts, \& Smith, 2010), in their review, Gupta et.al. (2018) defined OIRD as "respiratory rate less than $10 / \mathrm{min}$, and/or oxygen saturation less than $90 \%$, Naloxone administration, other respiratory events like apnea, hypopnea, respiratory failure, and excessive sedation and upper airway obstruction" (p. 111).

Opioid-induced sedation and respiratory depression (OSRD): The associated sedation or decreased level of consciousness that occurs with or without respiratory depression, which is considered a respiratory rate decreased from a patient's typical rate, 
after opioid administration (Pasero \& McCaffery, 2011). Often these events require the reversal of the opioid using Naloxone, an opioid antagonist (Pasero \& McCaffery, 2011).

Opioid-induced sedation: OSRD includes opioid-induced sedation, but the literature recognizes it as a precursor of respiratory depression and is described separately (Pasero \& McCaffery, 2011). Therefore, the early assessment of sedation is paramount in the detection of opioid adverse events.

Opioid-induced ventilatory impairment (OIVI): Caused by the depression of the central nervous system after opioid administration, which causes a decrease in respiratory drive and cognitive function and impacts the strength of the muscles in the airway (Macintyre, Loadsman, \& Scott, 2011).

Naloxone administration: Naloxone is an opioid antagonist and reverses the effects of opioids in the body, including the adverse effects of sedation and respiratory depression, and is often used in organizations as a quality indicator (Rosenfeld et al., 2016). It is a measurable outcome, although it may not encompass every instance of oversedation or respiratory depression because not every patient requires Naloxone for reversal as close monitoring and supplemental oxygen may suffice.

Practice factors: The risk factors that are dependent on the patient's surgery or circumstance and can change throughout their stay whether that be outpatient or inpatient, including the first 24 hours' postanesthesia, prolonged surgery, surgical type, concomitant use of sedating agents, large boluses of opioids, and/or patient-controlled analgesia (TJC, 2012). 
Pharmacogenetic factors: The way that genes impact reactions to medications is called pharmacogenetics (Chianta \& Guevara, 2014). There is a genetic variance in relation to opioids, and patients can be ultra-rapid metabolizers of opioids (Chianta \& Guevara, 2014). This can place a patient at risk for oversedation and respiratory depression; however, it is not a typical item known to patients because it requires specialized genetic testing (Chianta \& Guevara, 2014). When a patient does know they are an ultra-rapid metabolizer, it needs to be a part of the risk consideration for managing their pain, but because it is not a widespread test, it would not be a common comorbid condition to include in a simple screening tool. Gender is a demographic factor and is known to play a role in the pharmacogenetics because males and females experience pain and metabolize pain treatments differently (Packiasbapathy \& Sadhasivam, 2018). Brant et al. (2018) listed female sex as a significant predictor of OSRD, while Minkowitz et al. (2014) listed male sex as a risk factor. Because there is a known difference pharmacodynamically with conflicting evidence supporting each gender as a factor, I used gender in sampling and matching case-controls for this study.

\section{Assumptions}

In this quantitative study, I assumed the sample data of patients who received Naloxone to be the same as what were in the literature. Likewise, I assumed Naloxone administration for untoward events caused by opioids was an appropriate outcome measure for OSRD. The control was purposefully sampled to ensure it was like the case group. Another assumption was that the care of the patients in each group was the standard of care for each surgical procedure and the competency and knowledge of the 
nurses was also the same. This could not be controlled for, and therefore, an assumption was made about it. The items listed in the patient record were assumed to be an accurate representation of that patient and in the context of this study, trusting the accuracy of the patient record is imperative to complete the data extraction process. These assumptions were necessary to obtain access to a sample that likely represented the study population.

\section{Scope and Delimitations}

With this study, I addressed the gap that existed concerning the lack of a formalized tool to assess risk factors based on their prediction of OSRD. This study included a retrospective examination of charts of hospitalized patients that were older than 18 years old and had risk factors for OSRD. The records of patients who had received Naloxone for reversal of opioids were examined and compared with a similar group of patients who did not require Naloxone for reversal after opioid administration. Because the number of patients who receive Naloxone after opioid management is small compared to all hospitalized patients requiring pain treatment, prospectively analyzing and reviewing patient histories, then comparing them, had to be a longitudinal study. At the time of the study, the current rate of Naloxone administration at the facility where the sample was studied was $0.2 \%$ per 1,000 patient days, which would have required extensive time if I had conducted a prospective study; therefore, I did not perform a prospective review. I conducted a retrospective analysis of records from 2016 to 2019 of patients who received Naloxone and an equal sample of patients who did not receive Naloxone, which was feasible and appropriate to answer the research question. 
I analyzed each chart for the factors in the literature that place a patient at risk for OSRD. Once all the factors were retrieved and reviewed, statistical analysis was conducted to determine the factors that are most predictive of OSRD on admission the hospital setting. Outpatients were not included in this analysis because they represent a different population with different factors. In the outpatient community, the focus is on predicting the risk of addiction and abuse or overdose (Zedler, Saunders, Joyce, Vick \& Murrelle, 2018). Other theories and models related to nurse decision-making are available. The humanistic-intuitive model applies more to an emotional or personal context to decision-making (Muntean, 2012). While this approach is used to support decisions, in this research study, the easy-to-use tool that I designed with known factors did include emotional decision-making within its purpose. The information-processing model offers an analytical base to decision-making (Muntean, 2012). This model could inform a screening tool to support nursing decision-making but does not offer the application of intuition that is critical to nursing that the CCT supports.

\section{Limitations}

Due to the nature of a retrospective chart review, there were intrinsic limitations to the nonprospective, randomization of the cases. I matched the control sample for a surgical procedure or admitting diagnosis along with gender and randomization; however, this could have impacted the generalizability of the results. In this study, I used patient sample data from only one community-based hospital system, which was a limitation of the study. Another limitation was the potential that there may have been other patients who experienced OSRD that were not captured by the administration of 
Naloxone and instead were caught early enough to provide close monitoring and stimulation to avoid Naloxone. Due to my experience of caring for patients receiving opioids, it is necessary to note that additional measures were taken to ensure that my previous experience did not bias the results because as the sole investigator gathering data from patient records, I used a systematic and documented protocol to retrieve accurate data points.

\section{Significance}

Preventing OSRD in hospitalized patients is of utmost importance. While it is the responsibility of a clinician to use their judgment in dosing opioids that are ordered (Jarzyna et al., 2011), a risk scoring prediction tool to alert the clinicians to potential adverse outcomes would provide another line of defense in preventing a sentinel event. Knowing how the factors predict OSRD is a meaningful step in the process to address the problem and stop it before it occurs rather than assessing for sedation and administering Naloxone after.

Many tools have been previously developed to screen patients for other sentinel events like falls or pressure ulcers, and these serve to assist in clinical decision-making and warrant interventions to be taken to prevent such events (Kumar, Mahal, \& Rajappa, 2012). Alarmingly, the reporting of OSRD is thought to be less than the actual occurrence, with OSRD likely happening more often than the reported $0.5 \%$ of patients (TJC, 2012). This underreporting justifies the problem from a patient safety and quality standpoint. While organizations like TJC (2012) have suggested screening patients, there is no extant method of how to do this in a standardized way that improves patient 
outcomes rather than following an arbitrary recommendation. A screening tool based on risk factors that predict OSRD will assist clinicians and promote positive social change by supporting clinical decision-making to prevent harm to individuals from opioid administration. Better pain assessment that includes screening for risk factors that predict OSRD will also lead to a positive social change by preventing injury as severe as death for individuals seeking care and pain management within a hospital setting.

\section{Summary}

Providing safe, effective pain management is a primary concern in the acute care hospital setting. The best practice recommendation is to assess risk factors that predict OSRD before managing a patient with opioid treatment to ensure the safest care possible (TJC, 2012). However, there is no scoring tool that has been developed or tested that screens patients based on their prediction of OSRD on admission. A tool that assists nurses in assessing risk would support decision-making for appropriate interventions to manage risk and provide optimum pain management and monitoring. Conducting this study was the first step in creating a tool, based on predictors of OSRD, to support nurses and increase patient safety. In Chapter 2, I will describe the literature that provided a foundation and basis for this study. 
Chapter 2: Literature Review

\section{Introduction}

Pain assessment and management is an integral part of nursing care. Opioids are one of the interventions utilized to manage pain within the hospital setting, therefore, a full understanding of the risks of oversedation is essential. The opioid epidemic has revealed the negative side effects of opioids, and many people are becoming more aware of the dangers. In the hospital setting, opioid adverse drug events are described as OSRD and are reported by TJC (2012) as occurring in $0.5 \%$ of postsurgical patients, which is considered a low estimation. While identifying patients who may be at high risk for OSRD is suggested by the TJC, there is no standardized method for screening; instead, what exists is a list of potential risk factors for use in hospitals.

To identify patients at risk of OSRD, facilities can include the following risk factors in their policy and education for clinicians: (a) age greater than 55 years old, (b)

obesity, (c) untreated OSA, (d) preexisting pulmonary/cardiac disease or dysfunction, (e) major organ failure, (f) being a smoker, (g) ASA Patient Status Classification 3-5, (h) being opioid naïve, (i) opioid tolerance and are given more than their usual amount, (j) high dose PCA pump patients, (k) in the first 24 hours of opioid therapy, (l) the presence of thoracic and other large incisions that may interfere with adequate ventilation, (m) concomitant administration of sedating agents, and (n) patients who have already received Naloxone for opioid reversal (Jungquist et al., 2017). However, the development of a scoring tool based on predictors that screen patients for risk of OSRD is not available. Such a tool would allow nurses to assess risk and provide appropriate 
interventions to manage risks while providing optimum pain management and monitoring. A screening tool that encompasses and weighs the risk factors for OSRD appropriately can assist clinicians in pain assessment and management.

In this chapter, I review the search strategies used to identify literature on OSRD and describe the theoretical foundation that served as a guide for the study as well as the rationale. A synthesis of the literature on OSRD, including the methodologies of such studies, follows. Then, OSRD will be explained along with similar terms that describe the phenomena. Last, a complete description of the risk factors for OSRD with their supporting evidence with the historical factors or comorbidities are listed.

\section{Literature Search Strategy}

I used multiple databases to gather all the available evidence on the phenomena of OSRD for this literature search. The term opioid-induced sedation and respiratory depression was first searched in CINAHL and Medline databases combined, and 41 publications were found. Of these, the responses and letters to editors were excluded as well as those that were not pertinent to the phenomena upon review. The remaining nine articles included seven articles from various organizations that offer practice recommendations on the prevention and monitoring of OSRD. In exploring the medical subject heading terms of two pertinent studies, naloxone administration and dosage and hospitalized, was added to narrow down the search from 641 articles, mainly on the use of Naloxone outside of the hospital, to five that were about hospitalized patients. Out of these, I found two research articles that explored risk factors and predictors of Naloxone use for respiratory depression and oversedation in hospitalized patients. Next, the medical 
subject heading term, respiratory insufficiency chemically induced, was combined with opioid and searched, which led to 237 articles, many pertaining to outpatient prescription pain management. Several articles also pertained to those patients with chronic pain. Next, risk factors were added to be searched with respiratory insufficiency chemically induced, and 55 articles were found. When reviewed, I realized that pertinent articles from this group had already been located from previous searches.

Subsequently, I also completed the following searches with other terminology found in the literature. Opioid-induced ventilatory impairment was searched, returning seven total articles, two of which had already been located and reviewed; however, I also found an article explaining opioids, ventilation, and acute pain management which was added to the literature review. Opioid-induced respiratory depression was searched next, resulting in 333 initial articles found. Combining those search results with the term risk factors led to 39 articles. Twelve of these 39 articles were duplicates of those found previously, but three new studies were found, including a study of predictors of oversedation. A study looking at patients on opioids and their risk of respiratory arrest and another about evaluating the factors associated with Naloxone use for reversal of OIRD were also found. Opioid-induced sedation was searched next, initially revealing 108 articles; many of the articles dealt with the assessment of sedation postopioid administration. When risk factors were added to the search, the 10 resulting articles were duplicates of those already located.

In the CINAHL and Medline databases, I found 33 articles that pertained to the phenomena. Fifteen of these were research studies, four were based on closed claims, and 
11 were analyzing risk factors associated with OSRD. The remaining articles were practice recommendations on prevention and monitoring of OSRD.

In ProQuest, I searched both the Health and Medical and Nursing and Allied Health databases. The same terms were used as described previously, resulting in 897 articles, which was narrowed to 115 when the term risk factors was added to the search.

Of those, 16 were reviewed for content, three of which were research studies found in the previous search. The remaining 13 were reviews from nursing organizations: Three were from the pharmacology databases to assist in a better understanding of opioids and their action. I also searched PubMed using the search terms previously mentioned and combined. Out of 107 articles, 11 were pertinent to the phenomena and had already been identified in other databases.

Lastly, I completed a search of Google Scholar using the terms opioid-induced respiratory depression and risk factors, which initially produced 1,120 articles. When the search was narrowed to articles published after 2015, only one remained. Other articles that had cited this one remaining article were reviewed for further pertinent studies. I searched the other studies and reviewed the other articles that cited them for further pertinent research, locating two more studies to include in the literature review.

\section{Theoretical Foundation}

\section{Cognitive Continuum Theory (CCT)}

Assessing patients, including their risk for unplanned events, is crucial in making decisions for interventions. A simplified tool that assesses for risk factors of OSRD can assist in quick analysis and decision-making when managing pain with opioids. For this 
reason, I chose the CCT, introduced by Hammond (1988), as the theoretical foundation for this study. CCT is a theory that aids in understanding the judgment and decisionmaking process (Cader, et al., 2005).

Decision-making can almost be automatic in the day-to-day care of a patient, and there are often nursing skills that are carried out almost automatically, while others require more thought and deliberation. In $\mathrm{CCT}$, "the more structured a task is, the more analytically induced will be the decision-making mode" (Cader et al., 2005, p. 399). In other words, tools provide organization to support well-structured tasks. Hammond described tasks as being either well-structured or ill-structured; however, both types of tasks are relevant in nursing and defined in the CCT (Cader et al., 2005). A tool based on the factors that most predict OSRD could assist in providing more structure to the task of risk assessment.

Intuition is also crucial for clinical decision-making in practice, and the CCT describes it as well. Although a tool can assist and support decisions, nurses are accountable and able to use a complete patient assessment, along with clinical judgment, to provide care to patients. CCT includes the concept of quasirationality, which is the combination of two types of thought: intuitive and analytical (Dhami \& Thomson, 2012). Dhami and Thomson (2012) described quasirationality regarding managerial activity, but many of the descriptions transfer well to nursing. The ability to use critical thinking and judgment compares to using analytical information along with nursing intuition to make informed decisions. Successful managers must use both intuitive thinking along with analytical thinking (Dhami \& Thomson, 2012). In assessing and managing the risk of 
oversedation from opioids, nurses can use a tool to support analytical thinking along with their clinical judgment or intuition.

Nursing uses the CCT as a method for guiding analysis. For example, Offredy, Kendall, and Goodman (2007) described the theory to explore nurses' decision-making. They used case scenarios and interviews to assess how nurses made their prescribing decisions based on the CCT matrix (Offredy et al., 2008). Muir (2004) explained and described the debate in nursing as it relates to clinical decision-making. With some theories supporting analytical or intuitive strategies for decision-making in nursing, there is indeed a middle ground where the CCT offers acknowledgment to both types of thinking within the CCT matrix (Muir, 2004).

While a tool may only address the analytical thought spectrum, an understanding that nurses make decisions based on a combination of analytical thought, with the use of tools making them well structured, along with intuitive thinking. The CCT supported the overall purpose of developing a tool that assesses for risk factors. The risk factors for OSRD are what I used to address the research question of this study.

\section{Literature Review Related to Key Variables and Concepts}

The published literature provides a good understanding of the overall problem of OSRD and the risk factors for OSRD; however, there is a gap in an understanding of the risk factors that potentially predict OSRD due to lack of consensus regarding these factors. In this section, I discuss the methodologies and approach of published studies on the risk factors for OSRD, the concept of OSRD, and the historical factors that are most 
supported in the literature for predicting OSRD. Utilizing the risk factors in the literature and the results of this study, a screening tool was created for future research.

\section{Synthesis of Studies on the Predictors of OSRD}

In the following section, I review studies in the extant literature that sought to describe, identify, and/or predict the risk factors associated with OSRD. I found 10 published studies that analyzed and described OSRD in surgical and medical patients with varying risk factors (i.e., Brant et al., 2018; Gordon, 2005; Khelemsky et al., 2015; Lee et al., 2015; Pawasauskas et al., 2014; Ramachandran et al., 2011; Rosenfeld et al., 2016; Taylor et al., 2005; Weingarten et al., 2015; Weingarten et al., 2016). They are all nonexperimental and retrospective with both descriptive studies and case-control studies contributing to the current body of evidence in relation to the risk factors for OSRD.

A descriptive study is one that describes something that already exists and is not correlating or predicting relationships (Houser, 2015). Descriptive data are useful in providing knowledge on a sample, in this case, patients who had succumbed to OSRD. Khelemsky et al. (2015), Lee et al. (2015), and Rosenfeld et al. (2016) all completed retrospective descriptive studies that provided a good base for comparison of incidence and overall occurrences of OSRD within the hospitalized patient population. Rosenfeld et al. reported incidences of OSRD in surgical patients of 3.8 per 1,000 and 2.0 per1,000 in medical patients, determining surgical patients with continuous opioid infusions are at the highest risk for OSRD. Khelemsky et al. reported the incidence of OSRD at $0.1 \%$ out of 442,699 cases reviewed. Gupta et al. (2018) reported incidence in their descriptive analysis as ranging from $0.16 \%$ up to $0.53 \%$ when this data could be obtained and 
reported in their review of several studies. These studies support the TJC's (2012) estimation of $0.5 \%$ of surgical patients succumb to OSRD despite assumptions of underreporting in their publication.

One retrospective study described the overall incidence of solely nonsurgical patients (Herzig, Rotherberg, Cheung, Ngo, \& Marcantonio, 2014). These researchers reported the incidence of a respiratory event in their population as $0.97 \%$. This report was interesting because this number is higher than the reports on the surgical population alone. The studies that examined both surgical and medical cases of Naloxone administration, however, reported a higher occurrence of surgical patients over medical patients experiencing an episode. Brant et al. (2018) found 55\% of cases surgical with the remaining medical, and Rosenfeld et al. (2016) reported the incidence of medical Naloxone administrations at $0.2 \%$. More attention has been focused on surgical occurrences of OSRD, likely due to more patients being prescribed opioids for acute pain related to a surgical procedure.

A case-control analysis assists researchers to compare differences between individuals who experience an occurrence or outcome to individuals who do not (Houser, 2015). A case-control analysis is a nonexperimental study where there are intact groups that are carefully sampled to ensure quality of data to infer causality (Houser, 2015). The retrospective studies on the risk factors for OSRD have different case-control sampling methods and statistical analysis depending on their overall intent of the study.

In their case-control analysis examining Naloxone as a potential measure of quality and safety in pain management, Gordon and Pellino (2005) found differences in 
comorbidities between the Naloxone group and the control group, matched by surgical procedure and time. One difference was the age which revealed that patients who received Naloxone were older and received other concomitant central nervous system depressants. Their review supports the use of Naloxone as a measurement of OSRD as the group that received Naloxone reversal, had increased sedation, decreased respirations and oxygen saturation because of being managed with opioids for pain and processes could be put in place to address these concerns (Gordon \& Pellino, 2005). The use of Naloxone as an indicator of OSRD is supported in many of the other studies (Khelmesky, 2015, Pawasauskas, 2014, and Rosenfeld, 2016) and will be used in this research.

Ramachandran et al. (2011) completed a chi-square analysis in their case-control study to compare the risk factor prevalence of patients who had a postoperative critical respiratory event receiving opioids for pain, with the overall prevalence of postoperative patients. The analysis revealed an overall prevalence of congestive heart failure, postoperative acute renal failure, OSA, and dysrhythmias to be statistically significantly higher in the critical respiratory events group (Ramachandran, 2011). Likewise, Pawasauskas (2014) compared risk factors between a case group that received Naloxone for OSRD and control group that did not, matched based on the amount of opioids received. The results of their comparison showed renal disease, cardiac disease, respiratory disease, use of other sedating medications, and positive smoking status as statistically significant between the groups.

Regression analysis was used in multiple case-control studies to determine if a predictive relationship of risk factors existed in the following studies. Taylor et al.'s 
study (2005), utilized a matched case-control sampling design and the control was chosen randomly and then paired based on surgical procedure and diagnosis-related group. The logistic regression analysis found age, COPD, being on hydromorphone or having one of the following in their medical history: heart disease, congestive heart failure, respiratory disease, OSA, kidney disease, end-stage renal disease, and/or liver disease, were predictors for a respiratory event compared to a control group (Taylor, 2005). Weingarten et al. $(2015,2016)$ studied predictors in postoperative patients in a case-control study matched with two controls based on age, sex, and surgical procedure. They found OSA and events in the recovery room to be significant predictors of ORSD. In another analysis, Weingarten et al. (2016) reviewed Naloxone administration within the recovery room and matched the sample the same as their other analysis. This study reports OSA, an ASA score greater than 3, and high opioid requirements as predictors of a respiratory event (Weingarten et al., 2016). In Brant et al.’s (2018) study, a sample of hospitalized patients was obtained in reverse chronological order until the desired sample size was reached. This study utilized a regression model to determine if there were predictive relationships between risk factors and oversedation and respiratory depression events. They reported that female sex, renal disease, untreated OSA, receiving long-acting opioids, and as needed hydromorphone, were predictors of an episode of sedation or respiratory depression (Brant et al., 2018).

\section{Opioid-Induced Sedation and Respiratory Depression (OSRD)}

OSRD is the associated sedation or decreased level of consciousness that occurs with or without respiratory depression which is considered a respiratory rate decreased 
from a patient's typical rate, after opioid administration (Pasero \& McCaffery, 2011). Often these events require the reversal of the opioid using Naloxone, an opioid antagonist. Other terms published in the literature with similar meanings include opioidinduced sedation, OIRD, OIVI. These are defined following, but for this research are encompassed in this definition of OSRD.

Opioid-induced sedation. This is included in the definition of OSRD. However, it is also described separately in the literature as it is recognized as a precursor of respiratory depression (Pasero \& McCaffery, 2011). Therefore, the assessment of sedation is paramount in the detection of opioid adverse events early.

Opioid-induced respiratory depression (OIRD). OIRD is defined as the respiratory rate and depth which is reduced below the patient's baseline after an opioid (Pasero \& McCaffery, 2011). While rates at less than 8 breaths a minute are considered severe (Dahan et al., 2010), in their review, Gupta et.al. defined OIRD as "respiratory rate less than $10 / \mathrm{min}$, and/or oxygen saturation less than $90 \%$, Naloxone administration, other respiratory events like apnea, hypopnea, respiratory failure, and excessive sedation and upper airway obstruction" (2018, p.111). This definition defined their inclusion of a literature review determining which studies examined risk factors about the same outcome of OIRD.

Opioid-induced ventilatory impairment (OIVI). OIVI is explained as being caused by the depression of the central nervous system after opioid administration, which causes a decrease in respiratory drive and cognitive function and impacts the strength of the muscles in the airway (Macintyre et al., 2011). Acute pain management with opioids 
can be dangerous, especially if titrating solely to a pain score (Macintyre et al., 2011). OIVI is especially concerning for patients with OSA, where opioids compound concerns with airway that are already present (Macintyre et al., 2011)

\section{Predictors of Opioid-Induced Sedation and Respiratory Depression}

The following is the factors that will be included in the retrospective review to determine how they predict OSRD to OSRD to utilize in the tool development subsequent this analysis. There are many factors that place a patient at risk for OSRD. This study sought to determine which factors, present on admission, which are the historical risk factors.

Historical risk factors are those factors from the medical history. Many of these risk factors are described by TJC (2012) and the Society for Pain Management nursing practice recommendations (2011) as characteristics of patients who are at higher risk for OSRDs (The Joint Commission, 2012; Jarzyna et al., 2011). These factors include demographics and diagnosis that could be screened upon admission and would not necessarily change throughout the hospitalization and include: obstructive sleep apnea, obesity, age, pulmonary disease, cardiac disease, major organ failure, diabetes mellitus, opioid naïve, previous opioid use, ASA score, and others not fully supported in the literature like Smoking, gender and neurological disease.

Gender is a demographic factor and is known to play a role in the pharmacogenetics as males and females experience pain and metabolize pain treatments differently (Packiasbapathy \& Sadhasivam, 2018). Brant et al. (2018), list female sex as a significant predictor of OSRD while Minkowitz et al. (2014) list male sex as a risk factor. 
Because there is a known difference pharmacodynamically with conflicting evidence supporting each gender as a factor, gender will be used in sampling and matching casecontrols for this study.

OSA. OSA (also known as suspected sleep apnea or sleep-disordered breathing) is a factor which has been identified as a risk factor for an adverse opioid event. Jungquist et al., (2018) published a review of the literature on OSA and how to prevent OIRD. "Ninety percent of patients with sleep-disordered breathing are undiagnosed" (Jungquist et al., 2018, p. 602). This emphasizes the need to screen for undiagnosed sleep apnea. Tools exist to screen for this preoperatively (Meisenberg, Ness, Rao, Rhule, \& Ley, 2017). STOP-BANG is a tool developed to screen patients based on a simple questionnaire that alerts practitioners to a potential for undiagnosed OSA (Chung et al., 2012). It consists of nine factors or questions that accumulate to a risk score. If greater than three factors are present, there is a high risk of having undiagnosed OSA (Chung et al., 2012).

The recommended care for OSA surgical patients and patients who screen positive for OSA, but require surgery, include appropriate use of CPAP machines, monitoring post operatively and using an opioid sparing technique (Jungquist et al., 2018). Using opioid sparing techniques to prevent OSRD is recommended but is not always feasible in every surgical or medical population because the type of surgery may not allow for a regional block and monitoring is critical. Gupta et al. (2018) published a review of risk factors for OSRD and noted that even in small doses, opioids could cause OSRD in patients with OSA. Out of the 13 studies included in their review, four reported 
a statistically significant association of OSA. In their study of postoperative patients who suffered a critical respiratory event, not only was OSA listed as a risk factor, 3 of the 4 patients who died due to the critical respiratory event had a diagnosis of OSA. While it may be one of the strongest predictors, it is also associated with more severe episodes of OSRD, including death (Ramachandran et al., 2011).

Obesity. A BMI greater than $30 \mathrm{~kg} / \mathrm{m} 2$ is considered obese and morbid obesity is a BMI greater than $35 \mathrm{~kg} / \mathrm{m} 2$. Morbid obesity, per TJC, is one of the characteristics of patients who are at higher risk of OSRD, because they are at higher risk of having sleep apnea (The Joint Commission, 2012). Meisenberg, et al. (2017) completed a retrospective analysis of OSRD, and knowledge assessment of staff before implementing EMR bundles and found that one of the most common risk factors was obesity. In Khelemsky et al.'s (2015) study, the average BMI for those receiving Naloxone was $27 \mathrm{~kg} / \mathrm{m} 2$ as a part of their descriptive analysis.

Age. Meisenberg et al. (2017) found that age greater than 60 increased risk for OSRD. Gupta et al. (2018) described the mean age of studies in their synthesis of 13 different studies were greater than 60 years in eight of the studies, but three reported an age greater than 50 years as significant. Other retrospective reviews also list age as a contributing risk factor to OSRD (Felhofer, 2013, Minkowitz et al., 2014,). TJC delineated the "risk is 2.8 times higher for individuals aged 61-70, 5.4 times higher for age 71-80 and 8.7 times higher for those over 80" (TJC, 2012, p. 2) for OSRD. Therefore, the risk increases as age increases.

Pulmonary disease. Pawasauskas, et al. (2014), retrospectively analyzed 
patients' medical records who received Naloxone for OSRD and examined the patient characteristics that have been reported in the literature. Results revealed that patients who required Naloxone had 5.1 risk factors as opposed to their control that had 3.3 risk factors. One of the factors was respiratory disease. Dahan, Overdyk, Smith, Aarts, and Niesters (2013) published a systematic analysis of case reports of opioid-induced OIRD events, which showed a predictable pattern of respiratory disease that needs to be considered when administering opioids to chronic pain patients. In Phase 1 Naloxone reversals, matched for case-control analysis, pulmonary disease was one of the statistically significant factors contributing to the need for Naloxone reversal. While it may seem that pulmonary disease is synonymous with obstructive sleep apnea, they both add as a stand-alone factor in OSRD.

Cardiac disease. Cardiac disease or heart disease is a broad term which is a disease of the heart or blood vessels (American Heart Association, 2017). Ramachandran et al. (2011) concluded that congestive heart failure, cardiac dysrhythmias, coronary artery disease, and hypertension were all strongly associated OSRD in their retrospective study. This study likewise included these diagnoses as positive for cardiac disease.

Major organ failure. Renal failure is likely a risk factor due to the kidneys requirement to clear the opioids from the system and build up can be detrimental (Conway et al., 2006). Postoperative acute renal failure is one factor specifically thought to increase OSRD in patients on opioid therapy for acute surgical pain (Ramachandran et al., 2011). Liver failure is listed as a risk factor likely due to the first-pass metabolism of Morphine, a common opioid (Casey, 2018). 
Diabetes mellitus. DM is a factor not included in the TJC factors. Gupta et al. (2018) determined a history of DM was statistically significant in as a contributor to OSRD in two of the studies included in their review, and of high percentage in another. Therefore, DM was included as a factor in this study.

Previous opioid use. Patients who utilize opioids regularly at home are considered opioid-tolerant, meaning they have developed a pharmacological tolerance to the opioid and may require an increased dose (Cooney \& Broglio, 2017). Their pain can be challenging to manage in the hospital setting and may require frequent, increasing dosing of opioids (dose stacking), which can increase their risk for OIRD (Jungquist, 2017). For these patients, the pharmacists are often consulted to determine the patient 's equianalgesic dosing requirements and assist in planning for appropriate dosing and monitoring. Patients who have had no opioid use at home are at increased risk for OSRD due to the potentially increased sensitivity to opioids.

ASA score. The ASA scores is determined by considering the patient's comorbidities, medications and need for surgery before a patient receives anesthesia. ASA scores range from 1 to 6 with an allowance for E for emergencies. Higher scores mean a patient has more comorbid factors that make anesthesia riskier. ASA score greater than 3 was one of the three highest factors in Felhofer's study which examined which patients would be the best candidates for capnography monitoring post opioid administration based on their risk (Felhofer, 2013).

With an understanding of the methodologies and approaches of OSRD and the supporting literature for the concepts and variables for this study, the published literature 
provides an understanding of the overall problem of OSRD. There is a variety of risk factors for OSRD, some present on admission as a historical factor and others that arise during the hospitalization. Due to the lack of consensus regarding these factors, there remains a gap in the risk factors ability to predict OSRD.

\section{Summary}

OSRD is known in the literature as a serious safety concern and an understanding of the factors that predict OSRD is not fully known. In this chapter, I reviewed the search strategies performed utilizing the various terminology, synonyms that describe the phenomena of OSRD, and the factors that predict it. The CCT is the theoretical underpinning that supports nursing decision making and the foundation of understanding the predictability of the risk factors for OSRD to make better decisions in care. A review of the literature revealed several retrospective case-control studies that support the factors in predicting OSRD, however, the studies also had varying results. Although there were some factors that were supported by more than one study, there was not a consistent list recurring from each analysis. The factors in the literature that could be screened upon admission and would not necessarily change throughout the hospitalization include: OSA, obesity, age, pulmonary disease, cardiac disease, major organ failure, DM, and no previous opioid use. In Chapter 3, I will describe the methodology that was used in my study to answer the research question and further fill the gap that exists in medical literature. 
Chapter 3: Research Method

\section{Introduction}

While the risk factors for OSRD are available in the literature (e.g., Jarzyna et al., 2011, Jungquist et al., 2017, TJC, 2012), no scoring tool has been developed or tested for use in screening patients for OSRD risk. The purpose of this study was to create a prediction model based on published risk factors for OSRD. In Chapter 3, I describe the research design, methodology, threats to validity, and ethical considerations of the study.

\section{Research Design and Rationale}

In this study, I used the quantitative method and a retrospective, case-control design. The dependent variable was what distinguished the case from the control, the presence of OSRD. OSRD was measured by the administration of Naloxone for opioid reversal. The descriptive variables that were used in sample matching were the surgical procedure/admitting medical diagnosis and sex. The independent variables were risk factors for OSRD and had been identified in previous research. These risk factors were OSA, obesity, age, pulmonary disease, cardiac disease, major organ failure, DM, and no previous opioid exposure. This design allowed me to extract the risk factor data elements that were then statistically analyzed to address the research question concerning the prediction of OSRD.

I compared the risk factors for OSRD between those who experienced OSRD and those who did not, which allowed risk and prediction between whole groups to occur (see Houser, 2015). A case-control design was the most commonly used method to determine the risk factors for OSRD (Brant et al., 2018; Gordon \& Pellino, 2005; Pawasakas et al., 
2014; Ramachandran et al., 2011; Taylor et al., 2005; Weingarten \& Biphenyls, 2015; Weingarten et al., 2016). In the previous studies, groups were organized and selected by whether the patients experienced OSRD (or a similar event) or not. I used this same approach to determine which variables place the patient at the highest risk for developing OSRD. I used a retrospective approach to test the predictive model, although I acknowledged that the best determinant of reliability and validity is prospective. However, due to the limited number of patients who experience OSRD, a prospective study was beyond the scope of this study because it would have required an extended period to identify and obtain data (see Houser, 2015).

\section{Methodology}

\section{Population}

Medical records of hospitalized medical and surgical patients in an acute care hospital who were managed with opioids for pain was the target population for this study. The National Center for Health Statistics reported 35,061 hospital admissions for varying diagnoses in the United States in 2015 (Centers for Disease Control, 2017). There is not an estimation of how many patients are treated with opioids within these hospitalizations, but with a focus on adequate pain management, opioids are a foundation of treatment (Macintyre, 2011). For the purposes of this study, I used the term, pain management, synonymously with opioid use, and every patient should be screened for risk of OSRD upon admission (see TJC, 2012). 


\section{Sampling and Sampling Procedures}

I used convenience sampling to extract the cases that I needed for the case group and the control group. Group 1 was the case group of individuals who had OSRD, and Group 2 was the control group of patients who have not had ORSD. I accessed records using a report from the acute care hospital system where I conducted my research after obtaining approval to access the data. The outcome measure of naloxone administrations was already being collected at the facility and was entitled the "Narcan Report."

Inclusion criteria for Group 1. For Group 1, the inclusion criteria were patient records who received Narcan and were over 18 years of age. Patients from Group 1 were excluded if they received Naloxone but had not received opioids within the 4 hours before administration. This assumes they received Naloxone as a last resort when no other reason was present for decreased respirations that were easily identified.

Inclusion criteria for Group 2. For Group 2, the inclusion criteria were patient records who were also over 18 years of age and were matched to Group 1 based on their medical diagnosis on admission or surgical procedure type and gender. The other descriptor factors used in other studies, like age, were not used as matching in this study because age is also a known predictor of OSRD and pairing based on this would have excluded it as a potential predictor.

To determine the sample size, I used $\mathrm{G}^{*}$ power software. $\mathrm{G}^{*}$ power is a software that can calculate the effect size to determine the number of cases needed in a sample (Faul, Erdfelder, Buchner, \& Lang, 2009). I used a logistic regression, a priori power analysis with two tails, an odds ratio of 2.3 , power of .80 , with a binomial distribution to 
calculate a total sample size of 196 to run the analysis. In the acute care 100 to 199 bed hospital system where I collected data from, naloxone reversal was given at a decreasing rate of . $2 \%$ per 1,000 patient days in 2018 . Naloxone reversal was $.8 \%$ in 2014 . The most recent 100 patients that met the inclusion criteria were included for Group 1. Group 2 included 100 patients that were matched for a total of 200 patients, which exceeded the power analysis calculation.

\section{Procedures for Data Collection}

Because this was a retrospective chart review, it fell under Walden University's Institutional Review Board (IRB) policy approving exempt studies using existing data, if deidentified, for research purposes. I obtained IRB exempt status before any data extraction or collection procedure. After IRB approval from the hospital facility, I contacted the pharmacy liaison and information services department to receive the naloxone report and a report of discharged patient records that matched per sampling method for Group 2. To collect the data for Group 1, I extracted cases from the naloxone report and then retrieved specific information on the variable from the patient record. The information I recorded were the patient's age, gender, medical diagnosis or surgical procedure, and whether they had OSA, obesity, pulmonary disease, cardiac disease, major organ failure, DM, and/or no opioid exposure.

After I recorded the information for Group 1, I searched the discharged patient report, which was provided to me by the information services department at the hospital, to extract Group 2. I matched cases based on the patients' medical diagnosis or surgical procedure and gender. I then obtained the matched patient's age, and whether they had 
OSA, obesity, pulmonary disease, cardiac disease, major organ failure, DM, and no opioid exposure.

\section{Instrumentation and Operationalization of Constructs}

I reviewed patient records from an electronic medical record system utilizing an Excel worksheet. Patient records were the mainstay of sources used in the previously published articles related to risk factors for ORSD (i.e., Brant et al., 2018; Gordon \& Pellino, 2005; Pawasakas et al., 2014; Ramachandran et al., 2011; Taylor et al., 2005; Weingarten \& Biphenyls, 2015; Weingarten et al., 2016). Review of the charts allowed me to extract the descriptive and independent variables from the patient records using deidentified case numbers (see Appendix A).

The variables described in the study were operationalized as follows. The dependent variable was whether the patient succumbed to OSRD. I measured it by the administration of naloxone after opioid administration representing an episode of OSRD. This was a categorical variable of yes $=0$ and no $=1$ in the Excel spreadsheet. The two descriptive constructs were pulled and applied to match the case with the control. Surgical procedure or admitting medical diagnosis and the patient's sex were recorded and matched accordingly.

The independent variables, which were also descriptive, were those risk factors most evidenced in the literature that were present on admission. Both weight and age were recorded as continuous variables. Weight was documented as BMI in the patient record, and age was calculated per the date of birth in the record. The remaining independent variables were all categorical and were represented by a diagnosis present of 
yes $=0$ or no $=1$. Presence of OSA included a diagnosis of OSA or suspected OSA where a patient screened positive using the STOP-BANG score of 3 or more. STOPBANG is a tool developed to screen patients based on a simple questionnaire that alerts practitioners to a potential for undiagnosed OSA (Chung et al., 2012). The presence of pulmonary disease was determined via diagnosis of COPD, asthma, or being a current smoker. Determination of cardiac disease included the diagnosis of congestive heart failure, cardiac dysrhythmias, coronary artery disease, and hypertension. Major organ failure was identified with diagnosis of either renal or liver failure. DM was identified with a diagnosis also. Last, no opioid exposure was identified as yes if the patient was not currently taking an opioid prescription.

\section{Data Analysis Plan}

I used Statistical Package for the Social Sciences Version 24 to calculate a stepwise logistic regression to determine which risk factors are most predictive for OSRD. Logistic regression is used when there is more than one independent variable and the dependent variable is categorical (Warner, 2013). Logistic regression supports the ability to determine how well the independent variables predict the dependent variables and avoid confounding effects that may occur when analyzed separately (Sperendei, 2014). Therefore, logistic regression applied to my data analysis because this study was the first step in determining which variables should be included in the final prediction model and subsequent tool with multiple independent variables (see Sperendei, 2014). Another analysis that could have been used to determine the differences between the two groups would have been chi-square tests. Chi square was used by Ramachandran et al. (2011) to 
compare means of cases of two groups, and this determined if there were differences between the risk factors of those who have OSRD and those who do not, but it did not determine the predictability of the factors.

\section{Threats to Validity}

\section{Internal Validity}

One of the threats to the internal validity of this study was the use of secondary data because initial recording of the data cannot be controlled but is considered a highly accurate source of objective data (see Houser, 2015). To minimize selection effects of the data, I was the only researcher retrieving the secondary data in this study. I ensured that the same documents were used to document data for each patient record. The admission history, physician history, and physical assessment were the main areas that I searched for the risk factors in this study (see Houser, 2015). The definitions of the variables being retrieved remained constant in both the case and control groups and the data dictionary (see Appendix B), providing a definition of each item in clear view. Selection effects that can cause an unequal distribution between subjects were controlled through the matching of subjects (see Houser, 2015).

\section{External Validity}

Time and history effects due to the case sample being obtained from a report over the course of several years was an external validity threat because different interventions and care may have been occurring during various periods. To minimize these threats, I when possible, retrieved the control sample from a similar time frame as the matched case (see Houser, 2015). This was not always possible, so there is an external threat to the 
validity of the results because the interventions and actions in a certain timeframe may have been different than another, which limits the generalizability of the results (see Houser, 2015).

\section{Statistical Conclusion Validity}

To control for statistical conclusion validity, the design of the study needs to align with the question being answered by the statistical analysis (see Houser, 2015). In this study, I utilized a comparison group that was matched based on criteria to provide backing for the conclusion validity. I also clearly defined the variables of interest to help support the validity of the statistical analysis completed.

\section{Ethical Procedures}

Before any data collection, I obtained IRB approval from both Walden University and the hospital where the patient records were reviewed. The information services department of the hospital required IRB approval prior to assisting in the location of records that met the inclusion and exclusion criteria for study groups. The risk that this research presented was minimal because there were no actual human participants in the study, solely the records of patients previously cared for. Data were de-identified so that no information I collected could identify a patient after extraction. I kept the Excel spreadsheet in a password-protected folder only accessible by me. The de-identified data will be maintained for 5 years per Walden IRB policy. The study was conducted within my own work environment, which allowed for the opportunity to access the study sample; however, the policies and procedures of both IRBs in the accession of data were strictly followed. 


\section{Summary}

This retrospective, case-control study was the first step in the creation of a screening tool for risk of OSRD by determining how well the risk factors predict OSRD. This design was supported in the literature as appropriate for the variables presented and the subsequent analysis completed. The sample was precisely matched, and variables were meticulously extracted from the electronic medical record for accuracy to subsequently develop a tool with a sound foundation. A logistic regression model built with the independent variables that are most predictive of OSRD lent itself to the development of a tool based on the factors that most predict OSRD, excluding those with little odds of increasing OSRD. Data were de-identified and protection of human subjects was maintained through anonymity. I report the results of my study in Chapter 4. 
Chapter 4: Results

\section{Introduction}

An understanding of how risk factors predict OSRD can assist in the assessment of risk after opioid administration. The purpose of this quantitative study was to create a prediction model with the known risk factors for OSRD to determine how well they predict OSRD. In this chapter, I explain the data collection, statistical tests performed, and the results to answer the research question and hypotheses posed:

Research Question: What combination of factors most accurately predict the risk of OSRD in patients on admission to an acute care healthcare institution?

$H_{0}$ : The independent variables, which included the continuous variables of obesity, measured in BMI and age, and categorical variables as diagnosed yes or no, which included OSA, pulmonary disease, cardiac disease, major organ failure, diabetes mellitus, and no opioid exposure, do not predict the risk of OSRD.

$H_{\mathrm{A}}$ : The independent variables, which included the continuous variables of obesity, measured in BMI and age, and categorical variables as diagnosed yes or no, which included OSA, pulmonary disease, cardiac disease, major organ failure, diabetes mellitus, and no opioid exposure, predict the risk of OSRD.

\section{Data Collection}

After I obtained IRB approval (Approval Number 1442958-1), data were collected through a retrospective chart review from an acute care, nonprofit, community 
hospital system. Data from the first group of records were labeled as Group 1 and represented the case participants of records from patients who had received naloxone after an opioid administration. The most recent 100 records of patients admitted during 2019, 2018 and 2017 were included in the study. Records were reviewed for the following factors: surgical procedure or medical diagnosis, gender, BMI, age, OSA, pulmonary disease, cardiac disease, major organ failure, DM, and whether the patient was considered opioid naïve. Active smoking was added as criteria for pulmonary disease. Major organ failure was reexamined and named renal failure because, in this sample, renal failure was the only major organ failure noted. The factors described above were listed and organized on a spreadsheet with the corresponding data for each of the 100 patient records reviewed (see Appendix C). The data for each of the factors were extracted from each patient record. No individual identifiers were used in the study spreadsheet.

Once the collection of Group 1 data was completed, the surgical procedure or medical diagnosis and gender was shared with the hospital's information technology department who assisted me in obtaining data for Group 2, the control group. The medical diagnosis group was more difficult to obtain because patients had numerous admitting diagnoses. These were matched randomly by using the first patient, organized in reverse chronological order from May 2019 to January of 2019, who had either the same or the same category or type of diagnosis and gender. Once the records were selected each of the records was reviewed for gender, BMI, age, OSA, pulmonary disease, cardiac disease, renal failure, DM, and whether they were considered opioid 
naïve. Data were then listed and organized on a spreadsheet (see Appendix D). No individual identifiers were used in the study spreadsheet.

Table 1 shows the matched demographics of the 200 patient records included in the study. The sample included 136 total surgical patient records (i.e., 68 in Group 1 and 68 in Group 2) from various procedure types, including cardiac catheter procedure, general surgical procedures, gynecological procedures, neurosurgical procedures, orthopedic procedures, urological procedures and vascular procedures. There were 64 (i.e., 32 in Group 1 and 32 in Group 2) medical patient records from a variety of admitting diagnoses categorized as cardiac or respiratory diagnosis, gastrointestinal diagnosis, infection diagnosis, neurological diagnosis, and oncological diagnosis. The sample for Group 1 had a higher percentage of surgical (68\%) over medical (32\%) and female records $(72 \%)$ over male records $(28 \%)$. Therefore, the sample for Group 2 was matched to the Group 1 sample; the sample was randomly extracted based on the first surgical or medical procedure and gender, male or female, that corresponded from the report provided by the information technology department.

Table 1 denotes the matched demographics of the records included in the study. In the surgical procedure group ( $n=136,68 \%$ of total sample), the highest represented type was orthopedic ( $n=36,26 \%$ of surgical records), followed closely by vascular $(n=34$, $25 \%$ of surgical records) and general surgery ( $n=34,25 \%$ of surgical records) cases. Neurosurgical, urological, gynecological, and cardiac catheter procedures are represented but in small quantities. The medical diagnosis group ( $n=64,32 \%$ of total sample) is represented highest by those admitted for gastrointestinal issues ( $n=24,38 \%$ of medical 
records), such as small bowel obstruction and pancreatitis, followed closely by cardiac and respiratory diagnoses ( $n=22,34 \%$ of medical records), like shortness of breath. Infection, neurological, and oncological diagnosis are represented but in smaller quantity. The overall sample consisted of 144 (72\%) female patient records, this made up $73.5 \%$ of the surgical records $(n=100)$ and $68.7 \%$ of the medical records $(n=44)$. There were 66 (28\%) male patient records, which made up $26.5 \%$ of the surgical records and $31.2 \%$ of the medical records for the entire sample. 
Table 1

\section{Matched Demographics}

\begin{tabular}{|c|c|c|c|c|c|c|c|}
\hline \multirow[b]{2}{*}{ Factor } & \multirow[b]{2}{*}{$\begin{array}{c}\text { Total } n \\
(\%)\end{array}$} & \multicolumn{3}{|c|}{ Group 1} & \multicolumn{3}{|c|}{ Group 2} \\
\hline & & $\begin{array}{c}\text { Group } \\
n\end{array}$ & $\begin{array}{c}\% \\
\text { Female }\end{array}$ & $\begin{array}{c}\% \\
\text { Male }\end{array}$ & $\begin{array}{c}\text { Group } \\
n\end{array}$ & $\begin{array}{c}\% \\
\text { Female }\end{array}$ & $\begin{array}{c}\% \\
\text { Male }\end{array}$ \\
\hline$n$ & $200(100)$ & 100 & 72 & 28 & 100 & 72 & 28 \\
\hline Surgical & $136(68)$ & 68 & 73.5 & 26.5 & 68 & 73.5 & 26.5 \\
\hline Cardiac catheter & 2 & 1 & 100 & 0 & 1 & 100 & 0 \\
\hline General & 34 & 17 & 100 & 0 & 17 & 100 & 0 \\
\hline Gynecological & 6 & 3 & 100 & 0 & 3 & 100 & 0 \\
\hline Neurosurgical & 14 & 7 & 57 & 43 & 7 & 57 & 43 \\
\hline Orthopedic & 36 & 18 & 83 & 17 & 18 & 83 & 17 \\
\hline Urological & 10 & 5 & 40 & 60 & 5 & 40 & 60 \\
\hline Vascular & 34 & 17 & 47 & 53 & 17 & 47 & 53 \\
\hline Medical & $64(32)$ & 32 & 68.7 & 31.3 & 32 & 68.7 & 31.3 \\
\hline $\begin{array}{l}\text { Cardiac or } \\
\text { respiratory }\end{array}$ & 22 & 11 & 91 & 9 & 11 & 91 & 9 \\
\hline Gastrointestinal & 24 & 12 & 58.3 & 41.7 & 12 & 58.3 & 41.7 \\
\hline Infection & 8 & 4 & 25 & 75 & 4 & 25 & 75 \\
\hline Neurological & 6 & 3 & 66.7 & 33.3 & 3 & 66.7 & 33.3 \\
\hline Oncological & 4 & 2 & 50 & 50 & 2 & 50 & 50 \\
\hline
\end{tabular}


Medical records of hospitalized medical and surgical patients in an acute care hospital who were managed with opioids for pain was the target population for this study. The National Center for Health Statistics reported 35,061 hospital admissions for various diagnoses in the United States in 2015 (Centers for Disease Control, 2017). I assumed that there is a range of percentages of both surgical and medical admissions in hospitals nationwide, but the actual data are unknown. At this community-based healthcare system, there were 26,638 hospital admissions in 2018 and each hospital ranged from $31 \%$ to $46 \%$ surgical and $54 \%$ to $69 \%$ medical. The sample obtained for OSRD represented a higher surgical volume, likely due to higher amount of opioid prescriptions for acute pain.

\section{Study Results}

\section{Study Sample}

Table 2 denotes the descriptive data of the characteristics that represent the risk factors for OSRD. The table shows the total sample as well as the sample per group, subdivided into surgical and medical patients. Group 1 had a slightly lower BMI average than Group 2 at $28.3 \mathrm{~kg} / \mathrm{m} 2$ and $28.9 \mathrm{~kg} / \mathrm{m} 2$, respectively. The remaining characteristics of age, OSA, pulmonary disease (including being an active smoker), cardiac disease, renal failure, DM, and being opioid naïve were all higher in Group 1 than Group 2. The average age for Group 1 was 67.5 years and for Group 2 was 66 years. OSA was present in 30 patient records for Group 1 and two patient records for Group 2. Pulmonary disease was present in 37 patient records in Group 1 and 27 patient records in Group 2. Renal failure was present in 38 patient records in Group 1 and 25 patient records for Group 2. 
DM was present in 45 patient records in Group 1 and 38 records in Group 2. Lastly, 81 patients in Group 1 were opioid naïve where Group 2 had 79 opioid naïve patient records.

\section{Assumptions of Binary Logistic Regression}

1. The outcome variable is dichotomous. This is an assumption to ensure the dependent variable is binary, with only two options (Warner, 2013). In this study, OSRD was the dependent variable and was binary with either yes = being present or no $=$ not being present.

2. The scores must be statistically independent of each other, meaning they are not intercorrelated (Warner, 2013). Intercorrelated variables means the independent variables are increased because they have a relationship with each other separate from the outcome variable (Salmeron, Garcia, \& Garcia, 2018). To test this, I first analyzed each of the independent variables for multicollinearity by using a linear regression and analyzing each independent variable against the others. The variance inflation factor (VIF) estimates how much of the variance is inflated because of dependence on the other independent variable or predictor (Salmeron, et al., 2018). There is a varying opinion on acceptable VIF scores, a VIF score of less than 4 is considered acceptable in assuming there the predictor variables are not intercorrelated (Salmeron et al., 2018). When BMI was tested for collinearity, the VIF score for age was 1.159 , OSA was 1.086 , pulmonary disease was 1.079 , cardiac disease was 1.276 , renal disease was 1.147 , diabetes was 1.190 , and opioid naïve was 1.038. When age was tested for collinearity, the VIF scores for OSA was 1.150 , pulmonary disease was 1.072 , cardiac disease was 1.144 , renal 
disease was 1.164, diabetes was 1.280, opioid naïve was 1.023, and BMI was 1.183. When OSA was tested for collinearity, the VIF scores for pulmonary disease was 1.036 , cardiac disease was 1.285 , renal disease was 1.183 , diabetes was 1.278 , opioid naïve was 1.043, BMI was 1.191 , and age was 1.236 . When pulmonary disease was tested for collinearity, the VIF scores for cardiac disease was 1.292 , renal disease was 1.175 , diabetes was 1.267 , opioid naïve was 1.044 , BMI was 1.264 , age was 1.231 , and OSA was 1.106 . When cardiac disease was tested for collinearity, the VIF scores for renal disease was 1.136, diabetes was 1.237, opioid naïve was 1.049, BMI was 1.248, age was 1.095, OSA was 1.145, and pulmonary disease was 1.078 . When renal disease was tested for collinearity, the VIF scores for diabetes was 1.180, opioid naïve was 1.048, BMI was 1.227, age was 1.219 , OSA was 1.153 , pulmonary disease was 1.072 , and cardiac disease was 1.243. When diabetes was tested for collinearity, the VIF scores of opioid naïve was 1.04 , BMI was 1.177 , age was 1.24 , OSA was 1.151 , pulmonary disease was 1.068, cardiac disease was 1.251, and renal disease was 1.091. When opioid naïve was tested for collinearity, BMI was 1.252, age was 1.21, OSA was 1.147, pulmonary disease was 1.075 , cardiac disease was 1.294 , renal disease was 1.183, and diabetes was 1.269 . When put into the analysis individually, all the variables had VIF very close to 1 , which is the minimum value and all were less than 2. This meets the assumption that there is no intercorrelation between the predictor variables (see Appendix E). 
3. All relevant predictors are assumed to be present in the model (Warner, 2013). Based on evidence, the predictors included are those that were thought to predict OSRD and are present on admission.

4. Based on sampling methodology, the 200 records were assumed to be mutually exclusive, meaning cases of Group 1 are different patient records than cases in Group 2 (see Warner, 2013).

5. I used a logistic regression, a priori power analysis with two tails, an odds ratio of 2.3 , power of .80 , with a binomial distribution to calculate a total sample size of 196 was needed to run the analysis. The sample size I used in this study was large enough $(N=200)$.

\section{Research Question Results}

To answer the question fully, three different binary logistic regressions were performed. First, a binary logistic regression analysis was performed to determine the predictors of OSRD. The outcome variable of OSRD was coded as " 0 " for having OSRD and " 1 " for not having OSRD after opioid administration. Eight predictor variables were included. BMI and age as continuous variables and OSA, pulmonary disease, cardiac disease, renal failure, diabetes and opioid naïve. Each was coded as " 0 " if diagnosis was present or " 1 " if diagnosis not present. The binary logistic regression procedure was performed in Statistical Package for the Social Sciences Version 24. Data from 200 patient records, described above, were included in this analysis as described above.

A test of the full model against the null hypothesis model was statistically significant $X^{2}(1)=34.714 p=.000$. The strength of the association of the predictor 
variables to OSRD was weak with Cox \& Snell's $R^{2}$ of .159 and Nagelkerke's $=R^{2} .212$. Hosmer Lemeshow goodness of fit test was not statistically significant $p=.526$, therefore the model fits the data. The model predicted $62 \%$ of the individuals who had OSRD and $75 \%$ of the individuals who did not experience OSRD. Therefore 38 individuals were false positives and 25 false negatives. Overall model prediction occurrence was $68.5 \%$.

The output in Table 2 shows the raw score of the binary logistic regression coefficients, Wald statistics, and the estimated change in odds of OSRD for individuals with the presence of the diagnosis. The odds ratio $\operatorname{Exp}(B)$ of 1 indicates no change in odds of having OSRD. The results greater than 1 indicate a positive relationship based on BMI, age, or having the diagnosis or suspected diagnosis of OSA. BMI, age and obstructive sleep apnea are very close to 1 and therefore do not change the probability of having OSRD. Cardiac disease $(p=.207, \operatorname{Exp}(B)=1.675,95 \% C I, .752-3.732), \mathrm{DM}(p=$ $.272, \operatorname{Exp}(B)=1.485,95 \% C I, .733-3.008)$ and opioid naïve $(p=.202, \operatorname{Exp}(B)=1.653$, 95\% $C I, .763-3.581)$ patients are greater than 1 and increase the likelihood of having OSRD, but the results were not significant. Pulmonary disease and renal disease increased the odds of OSRD and are statistically significant with pulmonary having a $p$ value of $.001, \operatorname{Exp}(B)=3.706(95 \% C I, 1.747-7.862)$ and renal disease having a $p$ value of .004, $\operatorname{Exp}(B)=3.084(95 \% C I, 1.432-6.643)$. 
Table 2

Variables in the Equation

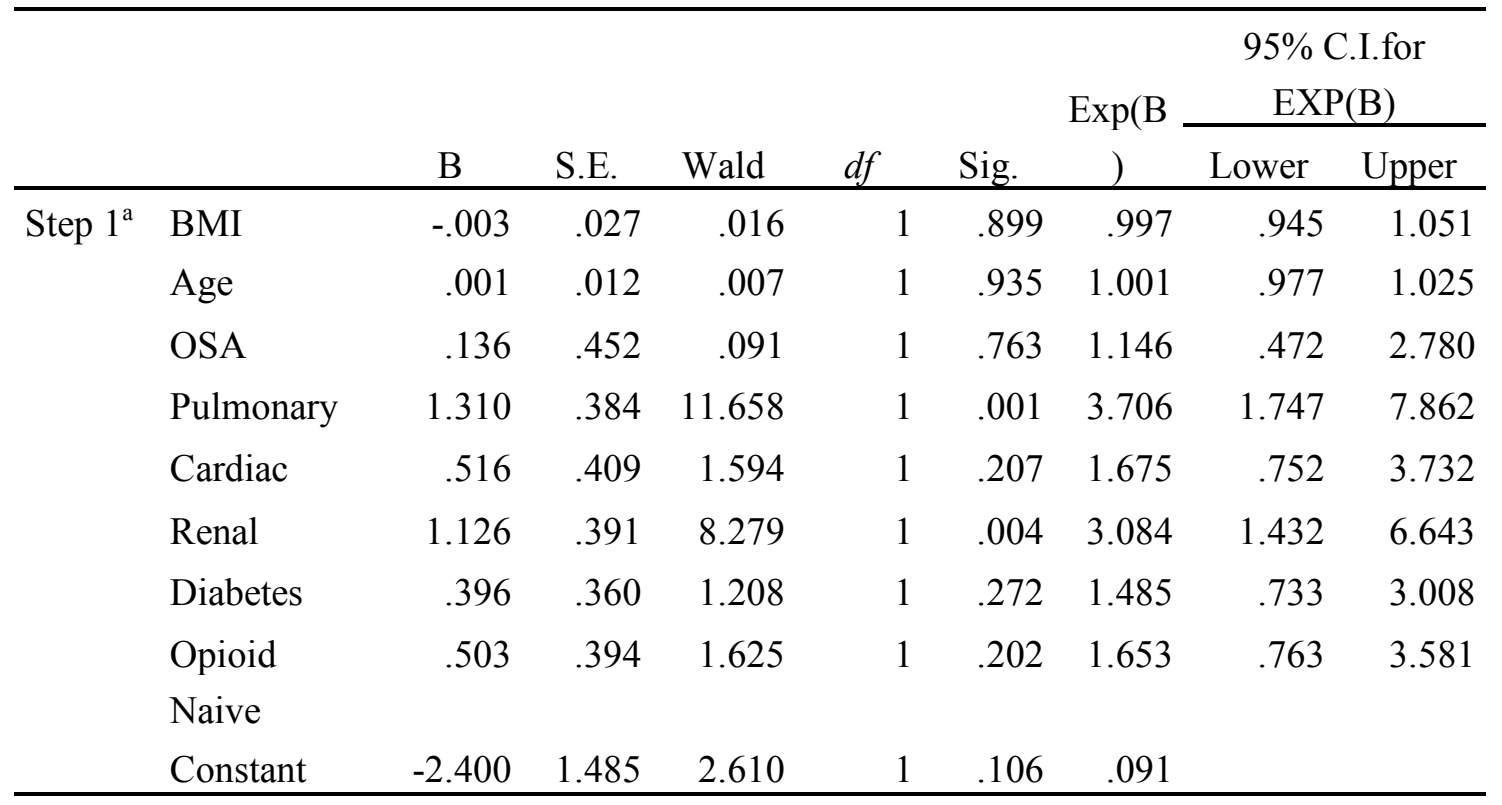

a. Variable(s) entered on step 1: BMI, Age, OSA, Pulmonary, Cardiac, Renal, Diabetes, Opioid Naive.

Next a forward stepwise binary logistic regression was completed to further analyze pulmonary disease and renal disease, the variables that were statistically significant, utilizing the same data and coding in the previous analysis. A test of the full model against the null hypothesis model was statistically significant in Step $1 X^{2}(1)=$ $15.417 p=.000$ and Step $2 X^{2}(1)=28.737 p=.000$. The strength of the association of the predictor variables to OSRD in step 1 was weak with a Cox $\&$ Snell's $R^{2}$ of .074 and Nagelkerke's $=R^{2} .099$. The strength of the association of the predictor variables to OSRD in Step 2 was weak with a Cox \& Snell's $R^{2}$ of .134 and Nagelkerke's $=R^{2} .178$. Hosmer Lemeshow goodness of fit test is not statistically significant $p=.999$, therefore the model fits the data. 
Table 3 summarizes the variables in the equation. Step 1 included renal disease only. The odds ratio $\operatorname{Exp}(B)$ was $3.765(95 \% C I, 1.88-7.539)$ and statistically significant, $p=.000$ therefore if renal disease is a single diagnosis, the patient has an increased likelihood of succumbing to OSRD after opioid administration. When renal disease was combined with pulmonary disease in Step 2, the presence of both diseases were statistically significant with $p=.000$. The odds ratio $\operatorname{Exp}(B)$ of pulmonary disease is $3.610(95 \% C I, 1.760-7.403)$ and renal disease is 3.767 (95\% $C I, 1.843-7.702)$, therefore with both diseases, the likelihood of OSRD after the administration of an opioid is even higher with the strength of the relationship increasing from a Nagelkerke's $=R^{2} .099$ in Step 1 to Nagelkerke's $=R^{2} .178$ in step 2 .

Table 3

Step-Wise Variables in the Equation

\begin{tabular}{|c|c|c|c|c|c|c|c|c|c|}
\hline & & \multirow[b]{2}{*}{$\mathrm{B}$} & \multirow[b]{2}{*}{ S.E. } & \multirow[b]{2}{*}{ Wald } & \multirow[b]{2}{*}{$d f$} & \multirow[b]{2}{*}{ Sig. } & \multirow[b]{2}{*}{$\operatorname{Exp}(B)$} & \multicolumn{2}{|c|}{ 95\% C.I.for $\operatorname{EXP}(B)$} \\
\hline & & & & & & & & Lower & Upper \\
\hline \multirow[t]{2}{*}{ Step $1^{\mathrm{a}}$} & Renal & 1.326 & .354 & 14.005 & 1 & .000 & 3.765 & 1.880 & 7.539 \\
\hline & Constant & -.999 & .313 & 10.201 & 1 & .001 & .368 & & \\
\hline \multirow[t]{3}{*}{ Step $2^{b}$} & Pulmonary & 1.284 & .366 & 12.275 & 1 & .000 & 3.610 & 1.760 & 7.403 \\
\hline & Renal & 1.326 & .365 & 13.215 & 1 & .000 & 3.767 & 1.843 & 7.702 \\
\hline & Constant & -1.971 & .440 & 20.051 & 1 & .000 & .139 & & \\
\hline
\end{tabular}

a. Variable(s) entered on step 1: Renal.

b. Variable(s) entered on step 2: Pulmonary.

A final binary logistic regression was completed with the variables in the previous procedures that increased odds of OSRD. BMI, age, and OSA were removed due to their $\operatorname{Exp}(B)$ not increasing the odds of OSRD in the first procedure. A test of the full model 
against the null hypothesis model was statistically significant $X^{2}(1)=34.560 p=.000$. The strength of the association of the predictor variables to OSRD was weak with Cox \& Snell's $R^{2}$ of .159 and Nagelkerke's $=R^{2} .212$. This final binary logistic regression has the same Nagelkerke's $\mathrm{R}^{2}$, despite having 3 variables removed from previous procedures. Hosmer Lemeshow goodness of fit test was not statistically significant $p=.899$, therefore the model fits the data.

Table 4 summarizes the variables included which were pulmonary disease $(p=$ $.000, \operatorname{Exp}(B), 3.781)$, renal disease $(p=.004, \operatorname{Exp}(B), 3.043)$, cardiac disease $(p=.169$, $\operatorname{Exp}(B), 1.688)$, diabetes $(p=.220, \operatorname{Exp}(B), 1.524)$, and opioid naïve $(p=.203, \operatorname{Exp}(B)$, 1.639). Pulmonary disease and renal disease remained the only statistically significant variables, $p=.000$ and $p=.004$, with increasing odds of OSRD at 3.781 and 3.043 which means every variable included increases the likelihood of OSRD after opioids, but pulmonary and renal disease have a higher likelihood of doing so with the Nagelkerke's = $R^{2} .212$, the same as the full model with all the variables included.

Table 4

Final Variables in the Equation

\begin{tabular}{llrrrrrrr}
\hline & & \multicolumn{1}{c}{ B } & \multicolumn{1}{c}{ S.E. } & \multicolumn{1}{c}{ Wald } & $d f$ & \multicolumn{1}{c}{ Sig. } & Exp(B) \\
\hline Step $1^{\mathrm{a}}$ & Pulmonary & 1.330 & .376 & 12.487 & 1 & .000 & 3.781 \\
& Renal & 1.113 & .383 & 8.460 & 1 & .004 & 3.043 \\
& Cardiac & .524 & .381 & 1.889 & 1 & .169 & 1.688 \\
& Diabetes & .421 & .344 & 1.503 & 1 & .220 & 1.524 \\
& Opioid Naive & .494 & .388 & 1.621 & 1 & .203 & 1.639 \\
& Constant & -2.337 & .499 & 21.945 & 1 & .000 & .097 \\
\hline
\end{tabular}

a. Variable(s) entered on step 1: Pulmonary, Renal, Cardiac, Diabetes, Opioid Naive. 
To answer the question posed, this final model combined with two statistically significant variables (pulmonary and renal) and those with increased odds of OSRD although not statistically significant (cardiac, diabetes, and opioid naïve) determined the factors that most accurately predict OSRD.

\section{Summary}

Based on the results of the analysis, the factors that most predict OSRD are the diagnosis of pulmonary disease, renal disease, cardiac disease, DM and being opioid naïve. The null hypothesis is rejected as the results revealed five factors that predict OSRD. Although only pulmonary disease and renal disease were statistically significant, the final model includes other factors that increase the odds of OSRD. These will be included in the development of a tool to predict OSRD. In Chapter 5, I will provide an interpretation of the results of the study, limitations, recommendations for future research, including a tool to be validated through expert opinion, implications, and conclusions. 
Chapter 5: Discussion, Conclusions, and Recommendations

\section{Introduction}

To determine the factors that predict OSRD on admission, I conducted a retrospective, case-control analysis was completed. The factors that most accurately predict OSRD are the presence of pulmonary disease, renal disease, cardiac disease, diabetes, and being opioid naïve. The null hypothesis was rejected because there are factors that predict OSRD. Although only pulmonary disease and renal disease were statistically significant, the final model includes other factors that increase the odds of OSRD. These factors will be included in the development of a tool to predict OSRD. In this chapter, I provide an interpretation of the results of the study, the limitations, and recommendations for future research. A tool will be presented for subsequent studies that will gain expert opinion on the factors and provide reliability and validity testing.

\section{Interpretation of Findings}

The published literature supported the overall problem of OSRD and the risk factors for OSRD (Brant et al., 2018; Gordon \& Pellino, 2005; TJC, 2012; Khelemsky et al., 2015; Lee et al., 2015; Pawasauskas et al., 2014; Ramachandran et al., 2011; Rosenfeld et al., 2016; Taylor et al., 2005; Weingarten \& Biphenyls, 2015; Weingarten et al., 2016). However, there was a gap in an understanding of the risk factors that potentially predict OSRD due to lack of consensus regarding these factors, specifically those present on admission. In this study, I sought to increase knowledge about the factors present on admission by understanding how well they predict OSRD. 


\section{Descriptive Statistics and the Literature}

The descriptive results of this study have many similarities and some differences to the sample populations of other studies in the literature that sought to better understand OSRD. The findings of this study confirmed results of studies that found a higher occurrence of OSRD in the surgical population over the medical population. Of the studies that examined both medical and surgical populations (i.e., Brant et al., 2018; Rosenfeld et al., 2015), the surgical population succumbed more frequently to OSRD. This is likely because of the higher doses required to manage acute pain in the surgical population. Previous studies examined only the surgical populations and reported the types of surgical procedures that most often had OSRD (see Gupta et al., 2018). The results of the current study confirmed other study demographics with patients undergoing orthopedic procedures as the highest number in the case group, those who succumbed to OSRD, comprising 18\% of the surgical sample (see Gupta et al., 2018; Lee et al., 2005; Weingarten et al., 2016). General surgical procedures comprised $17 \%$ of the surgical sample in this study. These proportions aligned with the literature, which stated the general surgical population may be at higher risk (Ramachandran et al., 2011; Rosenfeld et al., 2016; Taylor et al., 2005; Weingarten \& Biphenyls, 2015). I found that vascular procedures comprised $17 \%$ of the remaining surgical population of my sample. These procedures consisted of amputations and procedures to restore blood flow to extremities. Interestingly, these cases are not known to be in high volume in other literature; however, the comorbidities in this patient population are often diabetes and renal failure, which are known risk factors for OSRD (Gupta et al., 2018). Matching the cases with controls to 
include these types of procedures with their comorbidities adds to the validity of the results of this study because the procedure types with the common diagnoses of patients is important to determine the true predictability of the factors, not because the control did not match the case adequately.

The percentage of female patients in this study confirmed other retrospective reviews that also had a higher percentage of female patients who succumb to OSRD (Brant et al., 2018; Gupta et al., 2018; Khelemsky et al., 2015). Females composed 72\% of the case sample in this study, which was matched in the control group. This amount is slightly higher than what other studies reported (i.e., Brant et al., 2018; Gupta et al., 2018; Khelemsky et al., 2015). In studies that focused on OSA for predicting OSRD, the sample consisted of mostly males, which is a risk factor for OSA (Blake et al., 2008). Neither OSA nor obesity statistically predicted OSRD in my study, which may be because of the predominately female population; however, due to matching based on gender, predictability for gender was not determined.

Incidence of naloxone administration during the time of data collection (i.e., November 2016 to April 2019) ranged from 0.2\% to 0.6\%. Gupta et al. (2018) reported the incidence of OSRD in their descriptive analysis ranging from $0.16 \%$ up to $0.53 \%$. The studies in Gupta's analysis support the TJC (2012) estimation of $0.5 \%$ of surgical patients succumb to OSRD despite assumptions of underreporting of the incidence of OSRD to TJC. Therefore, when compared with other studies in the literature, the naloxone administration incidence at the facility under study was within a similar range of other reported samples. 


\section{Case-Control Result Comparison}

The results of the current study extend the knowledge in the field on the topic and confirm other the findings of similar studies that used the case-control design to predict the risk factors for OSRD. However, no other study had the same purpose as this study, which was to look only at factors present on admission and not throughout the hospitalization. Therefore, this is the first study to look only at the factors on admission.

Ramachandran et al. (2011) completed a chi-square analysis in a case-control study to compare the risk factor prevalence of patients who had a postoperative critical respiratory event receiving opioids for pain with the overall prevalence of the risk factors of postoperative patients. While the controls were different for the current study, Ramachandran et al. found renal failure to be a statistically significant risk factor. They also found statistically significant differences between risk factors of patients who had a postoperative critical event compared to the prevalence of these factors in their overall postoperative patients for congestive heart failure, dysrhythmias, and OSA. While the results of my study showed that the risk for OSRD increased with cardiac disease and OSA, there was not statistical significance. There may be a practical significance in including cardiac disease and OSA in a final tool, even without statistical significance, due to the confirmation of the increased odds of OSRD with the presence of cardiac disease and OSA.

Brant et al. (2018) examined the risk factors in case control groups and similarly found renal disease to be a statistically significant risk factor of OSRD; however, they also found female sex, untreated sleep apnea, receipt of long-acting oxycodone, and use 
of hydromorphone as predictors. Brant et al. did not include matching based on procedure or gender, which may be the reason for the differences in results. Brant et al. also examined patient factors throughout the hospital stay in relation to the opioids received, which is beneficial in planning management of pain for at risk patients.

Pawasauskas et al. (2014) compared risk factors between a case group that received Naloxone for OSRD and control group that did not, and matched cases based on the number of opioids received, which limited the understanding of the treatment potential similar to Brant et al.'s findings. However, Pawasauskas et al. showed that at different opioid consumption levels, some patients succumbed to OSRD more readily than others. The results of their comparison showed renal disease, cardiac disease, respiratory disease, use of other sedating medications, and positive smoking status as statistically significant between the groups. My study findings confirmed Pawasauskas et al.'s results of renal disease and respiratory disease (which includes positive smoking status) and the increased risk of cardiac disease, which although not statistically significant, did show an increased risk of OSRD.

Weingarten \& Biphenyls (2015) studied predictors in postoperative patients in a case-control matched study with two controls based on age, sex, and surgical procedure. They found OSA and events in the recovery room to be significant predictors of ORSD along with OSA, COPD, and DM. In another analysis, Weingarten et al. (2016) reviewed Naloxone administration within the recovery room and matched the sample the same as their other analysis. They reported OSA, an ASA score greater than 3, and high opioid requirements as predictors of a respiratory event as well as OSA, COPD, cardiac disease, 
and neurologic disease (Weingarten et al., 2016). In both Weingarten studies, the case and controls were matched similarly, although Weingarten only examined the postsurgical population in both studies. The results of my study confirmed characteristics from both of Weingarten's studies of COPD as statistically significant of predicting ORSD and the increased risk of ORSD if a patient has OSA and diabetes (Weingarten \& Biphenyls, 2015) as well as OSA and cardiac disease (Weingarten et al., 2016).

In relation to the $\mathrm{CCT}$, the results of this study assisted in the development of a tool that, once validated, will aid in the decision-making process of how much opioid to administer within a prescribed range and what monitoring may be used to prevent OSRD. The tool provides structure and organization to a task that assists in better decisionmaking in providing pain management with opioids (Cader et al., 2005). The theory also contains the concept of intuition, which is a clinician's critical thinking and judgment that influences prioritizing the management of pain with opioids and monitoring of a patient postopioid administration (Cader et al., 2005). Knowing a patient is at high risk based on a well-structured task, or in this case, screening tool, offers itself to better clinical decision-making.

The advantage of only examining risk factors present on admission substantiates the purpose of this study and narrowed the focus from all the risk factors in the literature throughout the continuum of care. This allowed for a magnified view of factors to be screened on admission in a simplified tool. Understanding the factors that place a patient at risk for ORSD admission may influence the way practitioners choose to manage pain throughout the hospitalization. Therefore, knowing oxycodone and hydromorphone 
increase the risk of OSRD (Brant et al., 2018), can assist in the development of an order set that avoids this type of opioid for pain management or patients that screen for risk of ORSD on admission.

\section{Limitations}

Per the power calculations, the sample size used in this study was sufficient; however, a larger sample size may have lent itself to greater generalizability of results. Matching based on surgical procedure and gender allowed for a more focused analysis. However, matching also limited the ability to determine if surgical procedure or gender predicted risk.

Retrospective studies are limited in their generalizability due to not being prospective or being able to randomize into the groups (Houser, 2013). Although I chose the control randomly to assist in rigor, retrospective data are not as strong as prospective data (see Houser, 2015). Retrospective data also limited the ability to accurately ascertain two variables: undiagnosed OSA and opioid naivety. It was difficult to examine undiagnosed OSA because the institution where I obtained the sample began screening regularly for undiagnosed sleep apnea in 2018 and only in the surgical population. This factor might not have been captured or conveyed due to the lack of standardized screening, especially in the medical patient population. This is likewise the case for determining if a patient is opioid naïve or not. Although the medications a patient was prescribed at home was obtained, it is difficult to know if a patient was consuming the opioids due to their "as needed" prescription. A detailed patient interview would be 
needed to accurately obtain the amount a patient was taking of an "as needed" prescription.

Although many surgical and medical patients were represented in this sample, it was only from one community-based hospital system. Therefore, the pool of patients was limited to the types of surgical procedures performed and medical diagnoses that are cared for in a single setting. This is another limiter of generalizability to all surgical and medical populations that are admitted across the country and world daily that may be different depending on their region or specialty hospital type.

Based on documentation of an opioid administration followed by sedation scores and respiratory rates, each patient in the case group was verified to need the administered naloxone which I used as the determination of OSRD in this study. However, it is possible that patients who received naloxone were having neurological or respiratory difficulties for reasons other than opioid administration, and therefore, the sample may not have captured all patients who had an OSRD event in the hospital during this time period. It is also likely that patients had OSRD but did not receive naloxone and so they were not captured in these data.

\section{Recommendations}

In their analysis Pawasakas et al (2014), determined "the overall number of risk factors, not necessarily the type of risk factor combinations played a large role in determining risk" (p. 749). Based on the results of my study, this is confirmed as the individuals in the case group, Group 1, had an average of 3.05 factors out of the six variables to include in the tool which are OSA, cardiac disease, opioid naïve, DM, 
pulmonary disease, and renal disease while the control group, Group 2, only had 2.16 on average. Based on this knowledge and the varying factors in all the samples, a tool that adds the number of risk factors and weights those with statistical significance higher to determine risk on admission may be the best option for a screening tool for OSRD. A draft of the Opioid-Induced Sedation and Respiratory Depression Screening Tool for future research includes; OSA, Cardiac disease, opioid naïve, DM counted as 1 point each and pulmonary disease and renal disease counted as 2 points each.

This retrospective analysis, along with the literature and theoretical underpinnings to provide structured tasks to assist in decision making, provided the background knowledge need to draft the tool explained above. Because age and BMI, did not show increased a patient's risk, they were excluded from the draft tool. OSA, although also not shown to increase risk in my data, but evidenced most frequently in other literature, will remain. Cardiac disease, DM, and opioid naïve factors all increase the odds of OSRD and although they were not statistically significant, they added to the overall model with pulmonary and renal disease which were statistically significant.

Initial testing of the tool drafted with these data was completed, adding the risk factors present in Group 1 and Group 2 and using the weights of 1 point if a diagnosis was present for OSA, cardiac disease, opioid naïve and DM and 2 points for pulmonary disease and renal disease. If the cutoff point is 3 , the number of false negatives in Group 1 is 26 and the number of false positives in Group 2 is 40 . Because determining risk for OSRD is the purpose of this tool, false positives where a patient is screened at risk even though they may not succumb to OSRD is a better outcome than a false negative. 
Therefore, based on the results of this study, the final tool will be reviewed and assessed by expert opinion and validated prospectively. Once experts agree with the factors, prospective analysis will validate the cut point for the tool and the usefulness of the tool in preventing OSRD in hospitalized patients. Further research on the practice factors that contribute to OSRD and the differences between the factors present on admission and those that occur during the hospitalization to determine if one outweighs the other in predicting OSRD should also occur.

\section{Implications}

Understanding the factors that predict OSRD to develop a screening tool could save lives in hospital institutions which is a positive social change. Patients have the right to remain free from harm in a hospital setting and appropriate tools that allow for screening can assist in such an endeavor. Prospective analysis for tool development would be difficult because OSRD occurs very infrequently. With the results of the current study and others in the literature, the factors present upon admission could be identified by screening to assist clinicians in appropriate management in both surgical and medical patients. Identifying risk highlights factors that might otherwise remain solely in a problem list upon a normal history retrieval from a patient.

\section{Conclusion}

With a better understanding of OSRD, the Opioid-Induced Sedation and Respiratory Depression Screening Tool could be the answer to those seeking ways to better screen for OSRD factors. The magnified view of factors only present on admission lends itself to a simple screen that when completed, will highlight the risk factors that 
may inadvertently be otherwise overlooked. Although there are more factors that lead to OSRD, this analysis captured those risk factors that can assist in determining care for the entire hospitalization therefore preventing over administration of opioids or lack of monitoring that can be tailored based on this screening tool. Certainly, clinical judgement is never replaced by a tool, but supporting decision making is essential to provide safe competent care in a sometimes-chaotic healthcare environment. 
References

American Heart Association. (2017). What is cardiovascular disease? Retrieved from http://www.heart.org/HEARTORG/Support/What-is-CardiovascularDisease_UCM_301852_Article.jsp\#.WRG85mnyt0w.

Brant, J. M., Buffington, C., Jurkovich, L. R., Karera, D., Stringer, L., Herbert, S., ... Mullette, E. J. (2018). Predictors of oversedation in hospitalized patients. American Journal of Health-System Pharmacy, 75(18), 1378-1385. https://doi.org/10.2146/ajhp170558

Cader, R., Campbell, S., \& Watson, D. (2005). Cognitive continuum theory. Journal of Advanced Nursing, 49(4). Retrieved from www.ncbi.nlm.nih.gov/pubmed/15701154

Casey, G. (2018). Understanding opioids. Kai Tiaki Nursing New Zealand, 24 (8), 20-24. Retrieved from https://www.ontario.ca/page/understanding-opioids

Centers for Medicare \& Medicaid Services. (2014). Requirements for hospital medication administration, particularly intravenous (IV) medications and post-operative care of patients receiving IV opioids. Baltimore, MA: Center for Clinical Standards and Quality/Survey \& Certification Group. Retrieved from https://www.cms.gov/Medicare/Provider-Enrollment-andCertification/SurveyCertificationGenInfo/Downloads/Survey-and-Cert-Letter-1415.pdf

Centers for Disease Control and Prevention. (2017). Hospital admission, average length of stay, outpatient visits, and outpatient srugery by type of owndership and size of 
hospital: United States, selected years 1975-2015 (Table 82). Retrieved from https://www.cdc.gov/nchs/data/hus/2017/082.pdf

Chianta, M., \& Guevara, M. (2014). Pharmacogenetics and pain management: An opportunity to advance personalized patient care. MLO: Medical Laboratory Observer, 46(2), 9. Retrieved from https:/www.mloonline.com/home/article/13006092/pharmacogenetics-and-pain-management-anopportunity-to-advance-personalized-patient-care

Chung, F., Subramanyam, R., Liao, P., Sasaki, E., Shapiro, C., \& Sun, Y. (2012). High STOP-Bang score indicates high probability of obstructive sleep apnea. British Journal of Anaesthesia, 108(5), 768-775. https://doi.org/10.1093/bja/aes022

Conway, B., Fogarty, D., Nelson, W., \& Doherty, C. (2006). Opiate toxicity in patients with renal failure. $B M J, 332,345-346$. http://dx.doi.org/10.1136/bmj.332.7537.345

Cooney, M., \& Broglio, K. (2017). Acute ain management in opioid-tolerant individuals. The Journal for Nurse Practitioners, 394-399. http://dx.doi.org/10.1016/j.nurpra.2017.04.016

Dahan, A., Aarts, L., \& Smith, T. W. (2010). Incidence, reversal, and prevention of opioid-induced respiratory depression. Anesthesiology, 112(1), 226-238. https://doi.org/10.1097/ALN.0b013e3181c38c25

Dahan, A., Overdyk, F., Smith, T., Aarts, L., \& Niesters, M. (2013). Pharmacovigilance: A review of opioid-induced respiratory depression in chronic pain patients. Pain Physician, 16(2), E85-E94. Retrieved from 
https://www.ncbi.nlm.nih.gov/books/NBK137930/

Dhami, M. K., \& Mumpower, J. L. (2018). Kenneth R. Hammond's contributions to the study of judgment and decision making. Judgment and Decision Making, 13(1), 122. Retrieved from http://journal.sjdm.org/17/171129a/jdm171129a.pdf .

Dhami, M. K., \& Thomson, M. E. (2012). On the relevance of cognitive continuum theory and quasirationality for understanding management judgment and decision making. European Management Journal, 30(4), 316-326. https://doi.org/10.1016/j.emj.2012.02.002

Faul, F., Erdfelder, E., Buchner, A., \& Lang, A.-G. (2009). Statistical power analyses using G*Power 3.1: Tests for correlation and regression analyses. Behavior Research Methods, 41, 1149-1160.

Fraser, M., \& Plescia, M. (2019). The opioid epidemic's prevention problem. American Journal Of Public Health, 109(2), 215-217. https://doi.org.10.2105/AJPH.2018.304859

Felhofer, K. (2013). Developing a respiratory depression scorecard for capnography monitoring. Innovations in Pharmacy, 4(3), 1-11. http://dx.doi.org/10.24926/iip.v4i3.310

Gordon, D. B., \& Pellino, T. A. (2005). Incidence and characteristics of naloxone use in postoperative pain management: A critical examination of naloxone use as a potential quality measure. Pain Management Nursing, 6(1), 30-36. https://doi.org/10.1016/j.pmn.2004.12.003

Gupta, K., Prasad, A., Nagappa, M., Wong, J., Abrahamyan, L., \& Chung, F. F. (2018). 
Risk factors for opioid-induced respiratory depression and failure to rescue: A review. Current Opinion in Anaesthesiology, 31(1), 110-119.

https://doi.org/10.1097/ACO.0000000000000541

Hammond, K. R. (1988) Judgement and decision making in dynamic tasks. Information and Decision Technologies, 14, 3-14.

Herzig, S., Rotherberg, M., Cheung, M., Ngo, L., \& Marcantonio, E. (2014). Opioid utilization and opioid-related adverse events in non-surgical patients in U.S. hospitals. Journal of Hospital Medicine, 9(2), 73-81.

https://doi.org/doi:10.1002/jhm.2102

Houser, J. (2013). Nursing research: Reading, using, and creating evidence. Burlington, MA: Jones and Bartlett Learning.

Jarzyna, D., Jungquist, C. R., Pasero, C., Willens, J. S., Nisbet, A., Oakes, L., ... Polomano, R. C. (2011). American Society for Pain Management nursing guidelines on monitoring for opioid-induced sedation and respiratory depression. Pain Management Nursing. https://doi.org/10.1016/j.pmn.2011.06.008

Jungquist, C. R., Smith, K., Wiltse Nicely, K. L., \& Polomano, R. C. (2017). Monitoring hospitalized adult patients for opioid-induced sedation and respiratory depression unintended advancing sedation and respiratory depression. American Journal of Nursing, 117(3), 27-35.

Khelemsky, Y., Kothari, R., Campbell, N., \& Farnad, S. (2015). Incidence and demographics of post-operative naloxone administration: A 13-year experience at a major tertiary teaching institution. Pain Physician, 18(5), E827-E829. 
Kumar, A., Mahal, R., \& Rajappa, T. (2012). Modified Delphi Technique: Validation of risk ssessment tool for the prediction of pressure ulcer. Ann Med Health Sci Res., 2(1), 36-41.

Lee, L. A., Caplan, R. A., Stephens, L. S., Posner, K. L., Terman, G. W., Voepel_Lewis, T., Domino, K. B. (2015). Postoperative opioid-induced respiratory depression. Pain Medicine, 3, 659-665. https://doi.org/10.1097/ALN.0000000000000564

Macintyre, P. E., Loadsman, J. A., \& Scott, D. A. (2011). Opioids, ventilation and acute pain management. Anaesthesia and Intensive Care, 39(4), 545-548.

McCaffery, M., \& Pasero, C. (2011). Pain assessment and pharmacologic management. St. Louis, MO: Mosby Elsevier.

Meisenberg, B., Ness, J., Rao, S., Rhule, J., \& Ley, C. (2017). Implementation of solutions to reduce opioid-induced oversedation and respiratory depression. American Journal of Health-System Pharmacy, 74(3), 162-169. https://doi.org/10.2146/ajhp160208

Minkowitz, H. S., Gruschkus, S. K., Shah, M., \& Raju, A. (2014). Adverse drug events among patients receiving postsurgical opioids in a large health system: Risk factors and outcomes. American Journal of Health-System Pharmacy, 71(18), 1556-1565. https://doi.org/10.2146/ajhp130031

Muir, N. (2004). Clinical decision-making: theory and practice. Nursing Standard, 47-54. http://dx.doi.org/10.7748/ns2004.05.18.36.47.c3614

Muntean, W. J. (2012). Nursing clinical decision-making : A literature review. Clinical Decision-Making, 1-24. 
Offredy, M., Kendall, S., \& Goodman, C. (2008). The use of cognitive continuum theory and patient scenarios to explore nurse prescribers' pharmacological knowledge and decision-making. International Journal of Nursing Studies, 45(6), 855-868. https://doi.org/10.1016/j.ijnurstu.2007.01.014

Packiasabapathy S, \& Sadhasivam S. (2018). Gender, genetics, and analgesia: Understanding the differences in response to pain relief. Journal of Pain Research, 2729.http://dx.doi.org/10.2147/JPR.S94650

Pawasauskas, J., Stevens, B., Youssef, R., \& Kelley, M. (2014). Predictors of naloxone use for respiratory depression and oversedation in hospitalized adults. American Journal of Health-System Pharmacy, 71(9), 746-750. https://doi.org/10.2146/ajhp130568

Ramachandran, S. K., Haider, N., Saran, K. A., Mathis, M., Kim, J., Morris, M., \& O’Reilly, M. (2011). Life-threatening critical respiratory events: A retrospective study of postoperative patients found unresponsive during analgesic therapy. Journal of Clinical Anesthesia, 23(3), 207-213. https://doi.org/10.1016/j.jclinane.2010.09.003

Rosenfeld, D. M., Betcher, J. A., Shah, R. A., Chang, Y. H. H., Cheng, M. R., Cubillo, E. I., \& Trentman, T. L. (2016). Findings of a naloxone database and its utilization to improve safety and education in a tertiary care medical center. Pain Practice, 16(3), 327-333. https://doi.org/10.1111/papr.12277

Salmeron, R., Garcia, C. B., \& Garcia, J. (2018). Variance inflation factor and condition number in multiple linear regression. Journal of Statistical Computation and 
Simulation, 88(12), 2365-2384. https://doi.org./10.1080/00949655.2018.1463376

Sperandei, S. (2014). Understanding logistic regression analysis. Biochemia Medica, 24(1), 12-18. https://doi.org/10.11613/BM.2014.003

Taylor, S., Kirton, O. C., Staff, I., \& Kozol, R. A. (2005). Postoperative day one: A high risk period for respiratory events. American Journal of Surgery, 190(5), 752-756. https://doi.org/10.1016/j.amjsurg.2005.07.015

The Joint Commission. (2012). Sentinel Event Alert \#49 - Safe use of opioids in hospitals. Retrieved from: https://www.jointcommission.org/sea_issue_49/

Weingarten, T., \& Biphenyls, C. P. (2015). Predictors of delayed postoperative respiratory depression assessed from naloxone administration. HHS Public Access, 91(2), 165-171. https://doi.org/10.1016/j.chemosphere.2012.12.037.Reactivity

Weingarten, T. N., Chong, E. Y., Schroeder, D. R., \& Sprung, J. (2016). Predictors and outcomes following naloxone administration during Phase I anesthesia recovery. Journal of Anesthesia, 30(1), 116-122. https://doi.org/10.1007/s00540-015-2082- 


\section{Appendix A: Data Spreadsheet}

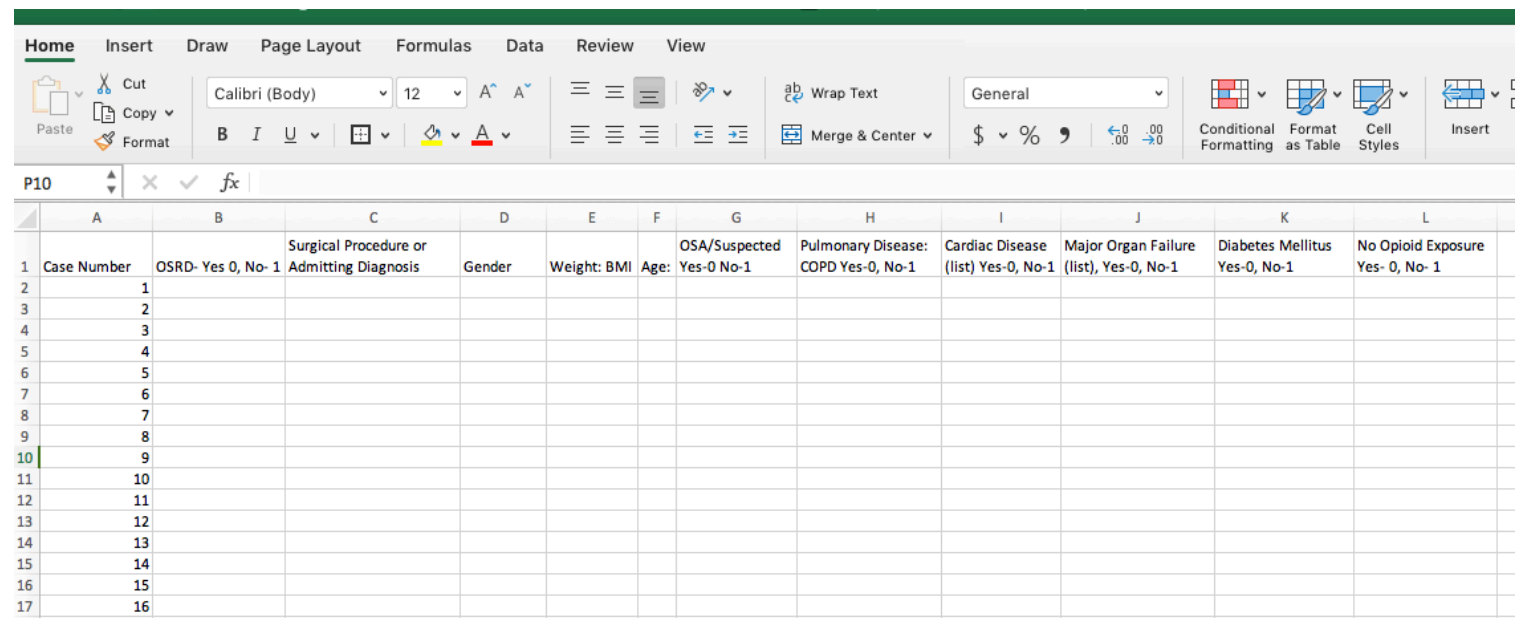


Appendix B: Data Dictionary

\begin{tabular}{|l|l|}
\hline \\
\hline 1
\end{tabular}


Appendix C: VIF Chart

Variance Inflation Factor (VIF) Chart

\begin{tabular}{lcccccccc}
\hline $\begin{array}{l}\text { Dependent } \\
\text { Variable }\end{array}$ & $\begin{array}{c}\text { Age } \\
\text { VIF }\end{array}$ & $\begin{array}{c}\text { BMI } \\
\text { VIF }\end{array}$ & $\begin{array}{c}\text { OSA } \\
\text { VIF }\end{array}$ & $\begin{array}{c}\text { Pulmonary } \\
\text { VIF }\end{array}$ & $\begin{array}{c}\text { Cardiac } \\
\text { VIF }\end{array}$ & $\begin{array}{c}\text { Renal } \\
\text { VIF }\end{array}$ & $\begin{array}{c}\text { Diabetes } \\
\text { VIF }\end{array}$ & $\begin{array}{c}\text { Opioid } \\
\text { Naïve } \\
\text { VIF }\end{array}$ \\
\hline Age & $\mathrm{X}$ & 1.183 & 1.150 & 1.072 & 1.144 & 1.164 & 1.280 & 1.023 \\
BMI & 1.159 & $\mathrm{X}$ & 1.086 & 1.079 & 1.276 & 1.147 & 1.19 & 1.038 \\
OSA & 1.236 & 1.191 & $\mathrm{X}$ & 1.036 & 1.285 & 1.183 & 1.278 & 1.043 \\
Pulmonary & 1.292 & 1.267 & 1.106 & $\mathrm{X}$ & 1.292 & 1.175 & 1.267 & 1.044 \\
Cardiac & 1.095 & 1.248 & 1.145 & 1.078 & $\mathrm{X}$ & 1.136 & 1.237 & 1.049 \\
Renal & 1.219 & 1.227 & 1.153 & 1.072 & 1.243 & $\mathrm{X}$ & 1.180 & 1.048 \\
Diabetes & 1.240 & 1.177 & 1.151 & 1.068 & 1.251 & 1.091 & $\mathrm{X}$ & 1.04 \\
Opioid & 1.21 & 1.252 & 1.147 & 1.075 & 1.294 & 1.183 & 1.269 & $\mathrm{X}$ \\
Naive & & & & & & & & \\
\hline
\end{tabular}

